\title{
Performance of UAV-assisted D2D Networks in the Finite Block-length Regime
}

\author{
Mehdi Monemi and Hina Tabassum, Senior Member, IEEE
}

\begin{abstract}
We develop a comprehensive framework to characterize and optimize the performance of a unmanned aerial vehicle (UAV)-assisted D2D network, where D2D transmissions underlay cellular transmissions. Different from conventional non-line-ofsight (NLoS) terrestrial transmissions, aerial transmissions are highly likely to experience line-of-sight (LoS). As such, characterizing the performance of mixed aerial-terrestrial networks with accurate fading models is critical to precise network performance characterization and resource optimization. We first characterize closed-form expressions for a variety of performance metrics such as frame decoding error probability (referred to as reliability), outage probability, and ergodic capacity of users. The terrestrial and aerial transmissions may experience either LoS Rician fading or NLoS Nakagami-m fading with a certain probability. Based on the derived expressions, we formulate a hierarchical bi-objective mixed-integer-nonlinear-programming (MINLP) problem to minimize the total transmit power of all users and maximize the aggregate throughput of D2D users subject to quality-of-service (QoS) measures (i.e., reliability and ergodic capacity) of cellular users. We model the proposed problem as a bi-partite one-tomany matching game. To solve this problem, we first obtain the optimal closed-form power allocations for each D2D and cellular user on any possible subchannel, and then incorporate them to devise efficient subchannel and power allocation algorithms. Complexity analysis of the proposed algorithms is presented. Numerical results verify the accuracy of our derived expressions and reveal the significance of aerial relays compared to ground relays in increasing the throughput of D2D pairs especially for distant D2D pairs.
\end{abstract}

Index Terms-Unmanned aerial vehicle (UAV), device-to-device (D2D), reliability, Rician, Nakagami-m, outage, subchannel and power allocation.

\section{INTRODUCTION}

Device-to-device (D2D) transmissions offer ubiquitous and ultra-reliable connectivity to diverse device types in $5 \mathrm{G}$ and beyond 5G (B5G) networks [1]. Among the two distinguished modes of D2D transmissions (i.e., overlay and underlay), the underlay mode where D2D transmissions coexist with the legacy cellular transmissions is relatively appealing to network operators due to its efficient spectrum utilization [2]. Typically, D2D communication facilitates direct communication between physically nearby devices, without the intervention of a base station (BS). To enable D2D communications, 3GPP introduced LTE-Direct protocol (also known as Proximitybased Services (ProSe)) in Release-12 [3]. Nevertheless, the applicability of ProSe remains limited in scenarios when the

This research was supported by a Discovery Grant funded by the Natural Sciences and Engineering Research Council of Canada. M. Monemi was a Visiting Researcher at the Dept. of Electrical Engineering and Computer Science, York University, Canada and is currently with the Dept. of Electrical and Computer Engineering, Salman Farsi University of Kazerun, Kazerun, Iran (email: monemi@kazerunsfu.ac.ir). H. Tabassum is with the Dept. of Electrical Engineering and Computer Science, York University, Canada. devices are outside the network coverage, far from each other, experience poor channel conditions, or create severe interference to cellular users. To address this limitation, relay-assisted D2D functionality has been introduced in 3GPP Release-13 [4]. Subsequently, a large body of the literature considered terrestrial relays with non-line-of-sight (NLoS) transmissions to instigate D2D communications [5], [6].

Along another note, unmanned-aerial-vehicles (UAV) based communication has emerged as a potential technology to complement terrestrial networks and will be an integral component of B5G wireless networks [7]-[11]. For example, in [9], the authors have proposed robust beam management and network self-healing mechanisms for millimeter wave (mm-wave) UAV mesh networks. In [10], a mm-wave distributed phased-arrays architecture and proof-of-concept (PoC) designs for mobile user equipment and UAVs were proposed. Also, in [11], the authors have studied the behaviour of mmWave air-to-ground channels by using two ray propagation model and employing ray tracing simulations for $28 \mathrm{GHz}$ and $60 \mathrm{GHz}$ frequencies.

Different from the terrestrial infrastructure experiencing mostly NLoS transmissions, UAVs can be deployed flexibly in three dimensions and can offer strong line-of-sight (LoS) connectivity. Nevertheless, in emerging 5G/B5G mixed aerial and terrestrial networks, we encounter statistically distinct LoS or NLoS fading channels and it is crucial to understand their impact on the network performance metrics and resource allocation schemes. To this end, this paper answers the fundamental questions such as (i) how to characterize key performance metrics such as outage probability, ergodic capacity, and decoding error probability of users in a UAVassisted D2D underlay network while accurately modeling mixed LoS/NLoS fading channels, (ii) how to use the derived expressions to efficiently manage network resources, and (iii) in which scenarios UAV-assisted D2D communications can be beneficial.

The impact of fading channel statistics on performance metrics can be computed by averaging over the entire distributions of the fading random variables. This can be done in two ways, i.e., (i) by computing the optimal solutions and resource allocations per fading channel realization. Then, solving the instantaneous optimization (resource allocation) problem for a large number of channel realizations and averaging over all channel realizations to compute the optimal solutions and performance metrics, or (ii) characterizing the statistically averaged performance metrics and then using them to formulate and solve the required optimization (resource allocation) problems. The latter approach enables us (1) to capture the impact of the random fading channel statistics on important performance metrics without solving the resource 
allocation problem for each channel realization and does not require averaging over all possible fading channel conditions, and (2) perfect knowledge of rapidly varying channel state information (CSI) is not needed.

\section{A. Background Work}

To date, a plethora of research works considered instantaneous optimization of the subchannel and power allocations of D2D users assuming either no fading [12]-[14] or Rayleigh fading [15], [16]. In [12], a successive convex optimization based resource allocation scheme was proposed to enhance the performance of D2D transmissions via UAV relays. In [13], the authors studied relay-assisted D2D communications in $\mathrm{mm}$ wave networks with full-duplex relays that are equipped with directional antennas and obtained a Pareto-optimal scheduling solution. In [14], the authors characterized the feasible region of interference in a D2D underlaid multi-cell cellular network and then devised efficient and reliable resource management schemes for network users. In [15], a two-sided resource allocation matching game is devised for decoupled uplinkdownlink multi-tier full-duplex networks under Rayleigh fading. In [16], a coalition game was established to maximize the sum-rate of underlaid D2D users in mm-wave networks.

Recently, few instantaneous optimization frameworks were developed considering Nakagami-m fading with shape factor $m$ for NLoS transmission links [17]-[19] and Rician fading with shape factor $K$ for LoS transmissions [20]-[22]. For example, in [17], by considering Nakagami-m fading channel, a user association and power control scheme was proposed for D2D underlaid cellular networks and then outage expressions have been derived. Assuming that the Shannon channel capacity is not reachable due to practical limitations, under the finite block-length regime, an ultra-reliable resource allocation scheme is devised in [18] for UAV-enabled networks wherein the channel is supposed to be Nakagami-m fading. In [20], an overlay/underlay mode selection and resource allocation algorithm was presented for mm-wave D2D networks under Rician fading channels. A coverage constrained scheduling scheme for UAV-assisted heterogeneous network (HetNet) was proposed in [21] under Rician fading channels. In [22], an energy-efficient power allocation scheme for UAV cognitive radio networks was proposed with Rician fading channels.

Nevertheless, in the aforementioned research works, the objective function and constraints are defined for each fading channel realization (which is assumed to be perfectly known) and, subsequently, the optimal solutions and resource allocations are computed per channel realization. The instantaneous optimization needs to be solved for a large number of channel realizations (assuming perfect CSI) to extract performance as a function of fading statistics.

Recently, a handful of research works exist that considered the outage probability expressions and ergodic rates in the design of resource allocation algorithms for HetNets [23][27]. For example, in [23], by considering no interference in an overlay D2D network, a minimum power scheduling scheme under Rician fading was proposed for full-duplex relay-assisted D2D communication. In [24], a throughput maximization problem with outage probability constraints was considered for UAV relay systems in the presence of Nakagami-m fading channels. In [25], a matching-game based subchannel and power allocation algorithm was proposed for a throughput maximization problem considering Rayleigh-faded NLoS signal and Rician faded LoS interference. In [26], by deriving the outage of users under Nakagami-m and Rayleigh fading channels, deployment cost efficiency was optimized in mm-wave networks.

\section{B. Motivation and Contributions}

We develop a comprehensive framework to characterize and optimize the performance of a UAV-assisted D2D network considering Nakagami-fading for NLoS and Rician fading for LoS transmission. Different from conventional NLoS terrestrial transmissions, aerial transmissions are highly likely to experience LoS. As such, characterizing the performance of mixed aerial-terrestrial networks with accurate fading models is critical to precise network performance optimization. To date, many research works rely on Rayleigh fading models for both the LoS and NLoS channels. While Rayleigh fading models are mathematically tractable, they are not accurate, especially for LoS modeling. Nakagami-m and Rician fading are considered as accurate ${ }^{1}$ for NLoS and LoS, respectively. Evidently, statistical characterization of the performance is even more challenging due to the coexistence of LoS Rician and NLoS Nakagami-m fading channels in the desired links and/or interfering links (especially when the desired signal experiences Rician LoS and the interfering signal experiences Nakagami-m NLoS or vice versa).

Furthermore, statistical modeling of the average performance metrics (such as decoding error probability, outage probability, and ergodic capacity) and applying them to devise efficient resource allocation schemes is another challenge. However, as mentioned earlier, this approach does not require to compute optimal solutions for each fading channel realizations (i.e., the optimization problem needs to be solved only once) and the instantaneous CSI is not needed.

To this end, the contributions of this paper are as follows:

- We consider a UAV-assisted D2D underlaid cellular network. Each D2D pair either selects direct or UAV-assisted relay transmission and all D2D transmission channels are shared with cellular users. Both the aerial and terrestrial transmissions experience LoS Rician fading or NLoS Nakagami-m fading depending on a specific criterion. We characterize the signal-to-interference (SIR) outage probability and ergodic capacity of cellular user transmissions, D2D transmissions, and UAV relay transmissions, considering Nakagami-m fading for NLoS and Rician fading for LoS channels.

- Under the finite block-length quasi-static regime and considering both LoS and NLoS interference scenarios, we derive analytic expressions for the frame decoding

\footnotetext{
${ }^{1}$ The models can capture a variety of channel conditions and empirical measurements. For example, $m=1$ in Nakagami-m distribution and $K=$ 0 in Rician distribution yield Rayleigh fading which is commonly used in terrestrial wireless networks.
} 
error probability of cellular users, which is also referred to as reliability [28]. We propose tight approximations to the decoding error probability and demonstrate through numerical results that the approximate expressions are in close agreement to the exact values. The derived closedform expressions for reliability can guarantee that the cellular users can always decode their block of (n bit) data (with a given probability).

- Based on the derived expressions, we formulate a hierarchical bi-objective optimization problem as a mixedinteger-nonlinear-program (MINLP) to minimize the total transmit power (the aggregate transmit power of UAVs as well as D2D and cellular users) leading to maximum aggregate throughput of D2D users, subject to QoS measures (i.e., reliability and ergodic capacity) of cellular users. The formulated problem allocates the power of cellular users as well as the power, subchannel and link-type (direct or relayed) for D2D pairs.

- We model the proposed problem as a bi-partite one-tomany matching game. In order to solve the problem, first we calculate the optimal closed form power allocation of each cellular user and D2D pair on each possible subchannel. Then, to assign the subchannel and link-type for D2D pairs, we first obtain the global optimal solution to the corresponding one-to-one matching game with no UAV relays, and then we extend the corresponding procedure to achieve the solution for one-to-many matching game scheduling scheme.

- Complexity analysis of the proposed algorithm is provided. Numerical results reveal that by choosing optimum height for UAVs, the performance measures of the proposed resource management schemes can be enhanced to a great extent, specially for distant D2D pairs.

The rest of the paper is organized as follows. In Section II, the system model and assumptions are presented. In Section III, we characterize the outage probability and channel capacity for cellular and D2D users. The frame decoding error probability of cellular users is characterized in Section IV. Resource allocation problem and solution are presented in Section V, and finally simulation results and conclusions are provided in Sections VI and VII, respectively.

\section{System Model AND Assumptions}

\section{A. Network Model}

Consider a network consisting $M^{c}$ cellular users and $M^{d}$ D2D pairs and a set of one or more rotary-wing UAV $\mathbb{S}^{2}$ with predefined spatial deployment. Let $\mathcal{M}^{c}$ be the set of cellular users whose minimum QoS requirements must always be guaranteed, and $\mathcal{M}^{d}$ be the set of D2D pairs who use available cellular resources, as long as minimum $\mathrm{QoS}$ requirements of all cellular users are preserved. D2D pairs with strong channels may choose direct connection, while those with poor direct

\footnotetext{
${ }^{2}$ Rotary-wing UAVs can hover over a certain location to ensure continuous coverage. Rotor blades in the rotary-wing UAV work exactly in the same way as a fixed wing, however constant aircraft forward movement is not needed to produce airflow over the blades. As such, a rotary-wing UAV can be considered as predeployed by the network operators for network performance assessment and resource allocation/optimization purposes.
}

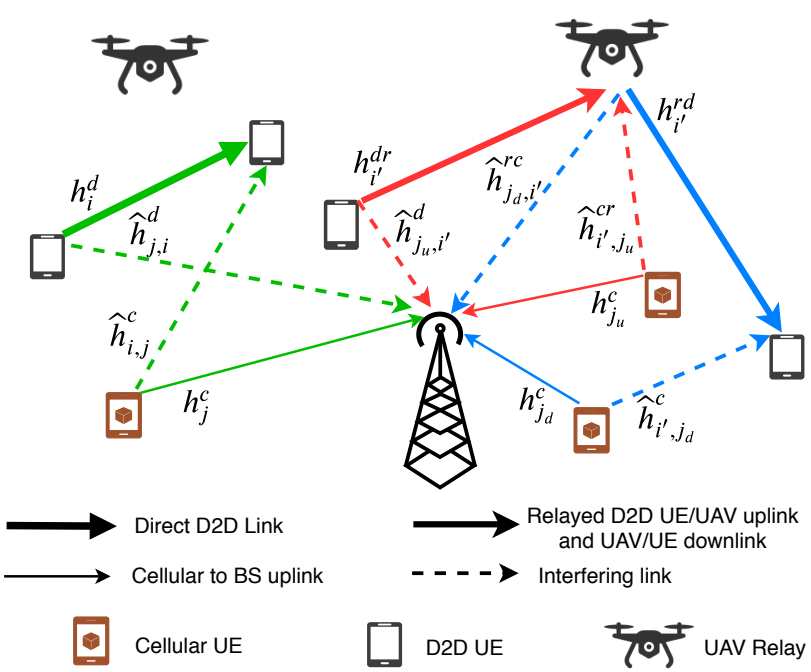

Fig. 1. Graphical illustration of D2D underlaid cellular network consisting of terrestrial BS, several cellular users, direct D2D and relayed D2D pairs. The subchannel allocated to cellular user $j$ is reused by D2D pair $i$ and corresponding links are shown in green color. The relayed D2D pair $i^{\prime}$ reuses the subchannels allocated to cellular users $j_{u}$ and $j_{d}$ in the uplink and downlink paths whose links are depicted in red and blue colors, respectively.

channel gain (i.e., those D2D pairs whose transmitters and receivers are rather far or potentially NLoS) may be served by the UAV relay. From now on, we will call the former and latter D2D pairs as direct D2D and relayed D2D pairs, respectively. It is seen in Fig. 1 that D2D pair $i \in \mathcal{M}^{d}$ and $i^{\prime} \in \mathcal{M}^{d}$ have established transmission through direct and relayed links, respectively. The relayed transmissions use decode-and-forward (DF) relaying mode.

\section{B. Channel Model}

Let $i \in \mathcal{M}^{d}$ be the direct D2D pair reusing the subchannel of cellular user $j \in \mathcal{M}^{c}$. Also, let $i^{\prime} \in \mathcal{M}^{d}$ be the relayed D2D pair reusing the subchannels of the cellular users $j_{u}$ and $j_{d}$ in the uplink and downlink paths, respectively (see Fig. 1). Let $h_{i}^{d}$ denotes the (power) channel gain between the transmitter and receiver of direct D2D pair $i \in \mathcal{M}^{d}$ and $h_{j}^{c}$ denotes the channel gain between the cellular user $j$ and ground BS. Also, let $h_{i^{\prime}}^{d r}$ and $h_{i^{\prime}}^{r d}$ denote, respectively, the relayed uplink and downlink channel gains for D2D pair $i^{\prime}$. For all relayed D2D pairs, we consider that the uplink and downlink channels are orthogonal to each other and may be shared with the subchannels of two different cellular users.

The interfering channel gain imposed from cellular user $j$ on the receiver of direct D2D pair $i$ is denoted by $\widehat{h}_{i, j}^{c}$, whereas that imposed from the transmitter of direct D2D pair $i$ to the receiver of cellular user $j$ (i.e., BS) is denoted by $\widehat{h}_{j, i}^{d}$. Also, for relayed D2D pair $i^{\prime}$, the interference imposed from cellular user $j_{u}$ on the UAV relay is denoted by $\widehat{h}_{i^{\prime}, j_{u}}^{c r}$ and the interference from the UAV relay to the receiver of cellular user $j_{d}$ (i.e., BS) is denoted by $\widehat{h}_{j_{d}, i^{\prime}}^{r c}$. We model any channel gain $h$ (whether desired or interfering link) as follows:

$$
h=x \mathbb{E}[h] \quad \text { where }
$$


TABLE I

FADING MODELS AND NOTATIONS CONSIDERED FOR DIFFERENT TRANSMISSION CHANNELS

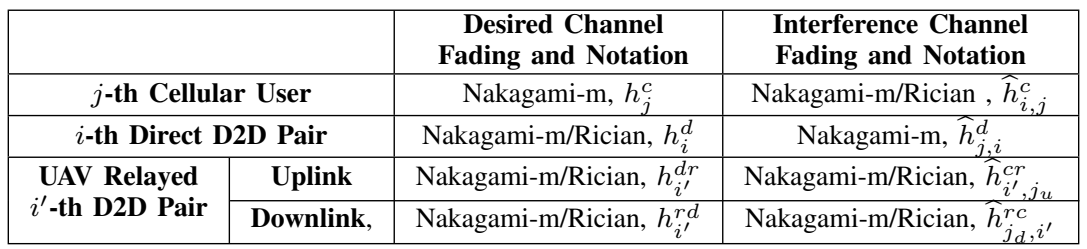

$$
\mathbb{E}[h](\mathrm{dB})=- \begin{cases}\mu_{L}+10 \beta_{L} \log _{10} d, & \text { for LoS link, } \\ \mu_{N}+10 \beta_{N} \log _{10} d, & \text { for NLoS link, }\end{cases}
$$

where $\mathbb{E}[h]$ is the expected value of $h$ considering the slow fading coefficients (i.e., path-loss and shadowing), and $x$ is unit mean random variable reflecting the fast fading coefficient which might not be exactly estimated. Also, $d$ is the distance of the link, $\mu_{L}$ and $\mu_{N}$ are frequency dependent components of the path loss, and $\beta_{L}$ and $\beta_{N}$ are the path loss exponents for the LoS and NLoS cases, respectively. We model the LoS and NLoS fading channels with Rician 3 distribution having shape factor $K$ and Nakagami-m distribution with shape factor $m$, respectively, as follows:

$$
\begin{aligned}
& f_{R}(x, K)=(K+1) e^{-K-(K+1) x} I_{0}(\sqrt{4 K(K+1) x}), \\
& f_{G}(x, m)=\frac{m^{m} x^{m-1}}{\Gamma(m)} e^{-m x},
\end{aligned}
$$

where $I_{0}($.$) and \Gamma($.$) are modified Bessel and gamma functions$ respectively. The fading models and notations considered for different aerial and terrestrial links are listed in Table 1. The LoS probability for aerial links is given by [30], [31]:

$$
p_{L}(\theta)=\frac{1}{1+C \exp (-B[\theta-C])},
$$

where $\theta$ is the angle between UAV and ground node, and $B$ and $C$ are constants related to environment. For transmissions between ground devices (i.e., transmission between each D2D transmitter and receiver, and interfering links from a cellular user to D2D receivers), we consider the 3GPP low altitude LoS probability model for urban environments as

$$
p_{L}(d)=\min \left(\frac{d_{1}}{d}, 1\right)\left(1-\exp \left(-\frac{d}{d_{2}}\right)\right)+\exp \left(-\frac{d}{d_{2}}\right) \text {, }
$$

where $d_{1}=18 \mathrm{~m}$ and $d_{2}=63 \mathrm{~m}$ [32].

\section{SINR Model for Direct Links}

Consider that direct D2D pair $i \in \mathcal{M}^{d}$ and cellular user $j \in \mathcal{M}^{c}$ are allocated same subchannel. The SINR at the D2D receiver is expressed as follows:

$$
\gamma_{i}^{d}=\frac{h_{i}^{d} p_{i}^{d}}{\mathcal{I}_{i, j}^{d}+\widehat{h}_{i, j}^{c} p_{j}^{c}},
$$

where $p_{i}^{d}$ and $p_{j}^{c}$ are the transmit powers of D2D pair $i$ and cellular user $j$ respectively, $\widehat{h}_{i, j}^{c} p_{j}^{c}$ is the dominant co-channel

\footnotetext{
${ }^{3}$ The approximation of Rician fading with Nakagami-m fading is generally not accurate for LoS channels [29], and thus in this work we have employed accurate Rician fading model for LoS channels.
}

intracell interference imposed from cellular user $j$ on D2D pair $i$, and $\mathcal{I}_{i, j}^{d}=\widetilde{\mathcal{I}}_{i, j}^{d}+\sigma^{2}$, in which $\sigma^{2}$ is the noise power, and $\widetilde{\mathcal{I}}_{i, j}^{d}$ is the interference imposed on D2D pair $i$ from all sources other than cellular user $j$ (which mainly results from the co-channel intercell interference due to frequency reuse in non-adjacent cells/sectors). Similarly, the SINR of cellular user $j$ is expressed as

$$
\gamma_{j}^{c}=\frac{h_{j}^{c} p_{j}^{c}}{\mathcal{I}_{j, i}^{c}+\widehat{h}_{j, i}^{d} p_{i}^{d}},
$$

where $\widehat{h}_{j, i}^{d} p_{i}^{d}$ is the dominant co-channel intracell interference imposed from D2D pair $i$ on cellular user $j$, and $\mathcal{I}_{j, i}^{c}=$ $\widetilde{\mathcal{I}}_{j, i}^{c}+\sigma^{2}$, in which $\widetilde{\mathcal{I}}_{j, i}^{c}$ is the interference imposed on cellular user $j$ from all sources other than D2D pair $i$. Note that the cellular user transmission can be interfered by either (i) the D2D transmitter in direct D2D relaying or (ii) either D2D transmitter or UAV relay in the relaying D2D link, and thus, as seen in Fig. 1 there is only interfering link for each subchannel at a time. Similarly, the D2D receiver or UAV relay will receive interference from a given cellular user transmission. Let $k$ be some (D2D or cellular) user and $k^{\prime}$ be its corresponding interfering user (if exists any). This way, if $k \in \mathcal{M}^{d}$, then we have $k^{\prime} \in \mathcal{M}^{c}$, and if $k \in \mathcal{M}^{c}$, then we have $k^{\prime} \in \mathcal{M}^{d}$. Now, in order to make notations simpler, we combine the SINRs in (6) (and also any other possible combinations for the relayed D2D pairs) as follows:

$$
\gamma_{k}=\frac{h_{k} p_{k}}{\mathcal{I}_{k, k^{\prime}}+\widehat{h}_{k, k^{\prime}} p_{k^{\prime}}},
$$

where $\gamma_{k}$ can be the SINR of any of the transmissions with D2D pair or cellular user, $h_{k}$ is the main channel gain of user $k$ and $\widehat{h}_{k, k^{\prime}}$ corresponds to the interfering channel gain imposed from user $k^{\prime}$ to user $k$, and finally $p_{k}$ and $p_{k^{\prime}}$ are transmit power levels of users $k$ and $k^{\prime}$ respectively. For example, if $k=i \in \mathcal{M}^{d}$ is a direct $\mathrm{D} 2 \mathrm{D}$ pair interfering with cellular user $j$, we have $h_{k}=h_{i}^{d}$, and $\widehat{h}_{k, k^{\prime}}=\widehat{h}_{i, j}^{c}$, and if $k=j \in \mathcal{M}^{c}$ is a cellular user, we have $h_{k}=h_{j}^{c}$, and $\widehat{h}_{k, k^{\prime}}=\widehat{h}_{j, i}^{d}$.

\section{SINR Model for Relayed Links}

The relayed transmissions use DF relaying mode. If D2D pair $i^{\prime} \in \mathcal{M}^{d}$ establishes a relayed link, the end-to-end SINR is the minimum of the SINR of the uplink and downlink [33], i.e.,

$$
\gamma_{i^{\prime}}^{d, r e l}=\min \left\{\frac{h_{i^{\prime}}^{d r} p_{i^{\prime}}^{d r}}{\mathcal{I}_{i^{\prime}, j_{u}}^{d}+\widehat{h}_{i^{\prime}, j_{u}}^{c r} p_{j_{u}}^{c}}, \frac{h_{i^{\prime}}^{r d} p_{i^{\prime}}^{r d}}{\mathcal{I}_{i^{\prime}, j_{d}}^{d}+\widehat{h}_{i^{\prime}, j_{d}}^{c} p_{j_{d}}^{c}}\right\} .
$$


where $j_{u}$ and $j_{d}$ are the cellular users whose subchannels are reused by relayed D2D pair $i^{\prime}$ in the uplink and downlink paths respectively, $p_{i^{\prime}}^{d r}$ is the transmit power of D2D transmitter to the relay, and $p_{i^{\prime}}^{r d}$ is the transmit power of relay to the receiver of D2D pair $i^{\prime}$. For brevity, we express all SINRs in the form of (8), and for some relayed D2D pair $k$, we rewrite (9) as $\gamma_{k}^{d, r e l}=\min \left\{\gamma_{k_{u}}, \gamma_{k_{d}}\right\}$, where $\gamma_{k_{u}}$ and $\gamma_{k_{d}}$ are the uplink and downlink SINRs which can be expressed from (8) as $\gamma_{k_{u}}=$ $\frac{h_{k_{u}} p_{k_{u}}}{\mathcal{I}_{k_{u}, k_{u}^{\prime}}+\widehat{h}_{k_{u}, k_{u}^{\prime}} p_{k_{u}^{\prime}}}, \gamma_{k_{d}}=\frac{h_{k_{d}} p_{k_{d}}}{\mathcal{I}_{k_{d}, k_{d}^{\prime}}+\widehat{h}_{k_{d}, k_{d}^{\prime}} p_{k_{d}^{\prime}}}$. For brevity, in the following sections, the notation $i$ may stand for either direct or relayed D2D pair.

\section{EXPRESSIONS OF OUTAGE PROBABILITY AND CHANNEL CAPACITY}

We characterize the outage probability for cellular transmissions, direct D2D, and relayed D2D transmissions considering interference limited regime. The expressions will be used to derive the ergodic capacity and the average frame decoding error probability of the cellular users.

\section{A. Outage Probability Analysis for a Typical Link}

Before deriving the outage probability, we ensure that the interference-limited assumption is always valid, i.e., we ensure that the interference in 8 is much higher than noise power, which leads to the inequality $\underline{p}_{k^{\prime}, k}<p_{k^{\prime}}$ where

$$
\underline{p}_{k^{\prime}, k}=\mathcal{I}_{k, k^{\prime}} \widetilde{K} / \mathbb{E}\left\{\widehat{h}_{k, k^{\prime}}\right\}
$$

in which $\widetilde{K} \gg 1$ is a constant. Therefore, the following outage probability derivations are valid when this inequality holds. For example, if direct D2D pair $i \in \mathcal{M}^{d}$ and cellular user $j \in \mathcal{M}^{c}$ share same subchannel, (6) and 10 correspond to $p_{i}^{d}>\underline{p}_{i, j}^{d}$ and $p_{j}^{c}>\underline{p}_{j, i}^{c}$ where $\underline{p}_{i, j}^{d}=\sigma^{2} \widetilde{K} / \mathbb{E}\left\{\widehat{h}_{j, i}^{d}\right\}$ and $\underline{p}_{j, i}^{c}=\sigma^{2} \widetilde{K} / \mathbb{E}\left\{\widehat{h}_{i, j}^{c}\right\}$. Similarly, for relayed D2D pairs, if $i \in \mathcal{M}^{d}$ reuses the subchannel of $j_{u} \in \mathcal{M}^{c}$ for the uplink and that of $j_{d} \in \mathcal{M}^{c}$ for the downlink, we must have $p_{i}^{d r}>\underline{p}_{i, j_{u}}^{d r}, p_{j_{u}}^{c}>\underline{p}_{j_{u}, i}^{c r}, p_{i}^{r d}>\underline{p}_{i, j_{d}}^{r d}$, and $p_{j_{d}}^{c}>\underline{p}_{j_{d}, i}^{c}$ where $\underline{p}_{i, j_{u}}^{d r}=\mathcal{I}_{j_{u}, i} \widetilde{K} / \mathbb{E}\left\{\widehat{h}_{j_{u}, i}^{d}\right\}, \underline{p}_{j_{u}, i}^{c r}=\mathcal{I}_{i, j_{u}} \widetilde{K} / \mathbb{E}\left\{\widehat{h}_{i, j_{u}}^{c r}\right\}$, $\underline{p}_{i, j_{d}}^{r d}=\mathcal{I}_{j_{d}, i} \widetilde{K} / \mathbb{E}\left\{\widehat{h}_{j_{d}, i}^{r c}\right\}$, and $\underline{p}_{j_{d}, i}^{c}=\mathcal{I}_{i, j_{d}} \widetilde{K} / \mathbb{E}\left\{\widehat{h}_{i, j_{d}}^{c}\right\}$. Let $y$ and $x$ denote respectively the unit mean fading variables of the main and interfering links $k$ and $k^{\prime}$. The outage probability function denoted by $O(\alpha)$ is expressed as

$$
O(\alpha)=\operatorname{Pr}\{y<\alpha x\}=\left\{\begin{array}{l}
O_{L, L}(\alpha), \text { if } k \operatorname{LoS}, k^{\prime} \operatorname{LoS}, \\
O_{L, N}(\alpha), \text { if } k \operatorname{LoS}, k^{\prime} \mathrm{NLoS}, \\
O_{N, L}(\alpha), \text { if } k \operatorname{NLoS}, k^{\prime} \operatorname{LoS}, \\
O_{N, N}(\alpha), \text { if } k \mathrm{NLoS}, k^{\prime} \mathrm{NLoS},
\end{array}\right.
$$

in which the outage representations of $O_{L, L}(\alpha), O_{L, N}(\alpha)$, $O_{N, L}(\alpha)$, and $O_{N, N}(\alpha)$ are studied later in this section. The outage probability value of user $k$ is then obtained as

$$
\operatorname{Pr}\left\{\gamma_{k}<\widehat{\gamma}_{k}\right\}=\operatorname{Pr}\left\{y<\zeta_{k, k^{\prime}} \widehat{\gamma}_{k} x\right\}=\left.O(\alpha)\right|_{\alpha=\zeta_{k, k^{\prime}} \widehat{\gamma}_{k}}
$$

where $\widehat{\gamma}_{k}$ is target-SINR of user $k$, and

$$
\zeta_{k, k^{\prime}}=p_{k^{\prime}} \mathbb{E}\left[\widehat{h}_{k, k^{\prime}}\right] / p_{k} \mathbb{E}\left[h_{k}\right] .
$$

If the link relating to the main user $k$ and that relating to the interfering user $k^{\prime}$ are both LoS with Rician shape factors
$K$ and $K^{\prime}$, the outage probability expressed in (11a) can be obtained as follows [34]: where ${ }_{2} F_{1}(\cdot)$ is the Gauss's hypergeometric function. To obtain closed-form expressions for $(11 \mathrm{~b}-11 \mathrm{~d})$, we consider the shape factor of Nakagami-m fading to be integer.

Lemma 1. For the case when the links of users $k$ and $k^{\prime}$ are LoS and NLoS with shape factors $K$ and $m$ respectively, the outage probability expression in $11 \mathrm{~b}$ is obtained as

$$
\begin{aligned}
O_{L, N}(\alpha) & =1-\frac{m^{m} e^{-K}}{\Gamma(m)(m+\alpha(K+1))^{m}} \times \\
& \frac{d^{m-1}}{d \theta^{m-1}}\left[\frac{\theta^{m-1}}{1-\theta}\left(e^{K}-\theta e^{K \theta}\right)\right]_{\theta=\frac{\alpha(K+1)}{m+\alpha(K+1)}}
\end{aligned} .
$$

\section{Proof. See Appendix A}

Once $O_{L, N}(\alpha)$ is obtained through $(15), O_{N, L}(\alpha)$ can be obtained from the following.

Lemma 2. For the case when the links of users $k$ and $k^{\prime}$ are $N L o S$ and LoS with shape factors $m$ and $K$ respectively, the outage probability expression in (11c) is obtained as follows:

$$
O_{N, L}(\alpha)=1-O_{L, N}(1 / \alpha) \text {. }
$$

Finally, we derive the outage probability of $O_{N, N}(\alpha)$ as follows.

Lemma 3. Let the links of users $k$ and $k^{\prime}$ both be NLoS with Nakagami-m shape factors $m$ and $m^{\prime}$ respectively. The outage probability expression in $(11 \mathrm{~d})$ is obtained as

$$
O_{N, N}(\alpha)=1-\frac{m^{\prime m^{\prime}}}{\Gamma\left(m^{\prime}\right)} \sum_{k=0}^{m-1} \frac{(m \alpha)^{k} \Gamma\left(k+m^{\prime}\right)}{k !\left(m^{\prime}+m \alpha\right)^{k+m^{\prime}}} .
$$

\section{Proof. See Appendix B.}

1) Outage Probability for Cellular Users: As discussed before, the desired links between the cellular users and their serving BS and interfering links from D2D transmitters to the cellular BS follow Nakagami-m fading channels. However, for relayed D2D links, the interfering link from UAV to cellular BS may follow LoS Rician fading, therefore in general, the outage probability of a cellular user $j$ is obtained as $\left.O^{c}(\alpha)\right|_{\alpha=\zeta_{j, i} \widehat{\gamma}_{j}}$ where

$$
O^{c}(\alpha)= \begin{cases}O_{N, L}(\alpha), & \text { for } \operatorname{LoS} \text { interference, } \\ O_{N, N}(\alpha), & \text { for NLoS interference. }\end{cases}
$$

2) Outage Probability for Direct D2D Pairs: Suppose some direct D2D pair $i$ reuses the subchannel of cellular user $j$. Depending on the distance between D2D transmitter and receiver and also the distance between the D2D receiver and the corresponding interfering cellular user, there exists positive probability values for LoS and NLoS links for each of the desired and interfering links. Therefore, the outage in 11 may have any of the four possible expressions, and thus the outage 
value is obtained by $\left.O^{d, d i r}(\alpha)\right|_{\alpha=\zeta_{i, j} \widehat{\gamma}_{i}}$, where $O^{d, d i r}(\alpha)$ is obtained by

$$
O^{d, d i r}(\alpha)= \begin{cases}O_{L, L}(\alpha), & \text { if } i \operatorname{LoS}, j \operatorname{LoS}, \\ O_{L, N}(\alpha), & \text { if } i \operatorname{LoS}, j \mathrm{NLoS}, \\ O_{N, L}(\alpha), & \text { if } i \mathrm{NLoS}, j \mathrm{LoS}, \\ O_{N, N}(\alpha), & \text { if } i \mathrm{NLoS}, j \mathrm{NLoS}\end{cases}
$$

3) Outage Probability for Relayed D2D Pairs: Now consider a relay-assisted D2D link. Depending on the the relative positions of the UAV, cellular BS, and the transmitter and receiver of the D2D pair, the desired and interfering signals in both the uplink and downlink may follow LoS or NLoS fading. The following lemma can be easily verified.

Lemma 4. Consider the relayed D2D pair $i \in \mathcal{M}^{d}$ where $U A V$ receives data from the $D 2 D$ transmitter on the uplink subchannel shared with cellular user $j_{u} \in \mathcal{M}^{c}$ and relays data to the corresponding D2D receiver on the downlink subchannel shared with cellular user $j_{d} \in \mathcal{M}^{c}$. The outage probability can thus be obtained as $\left.O^{d, r e l}\left(\alpha_{u}, \alpha_{d}\right)\right|_{\alpha_{u}=\zeta_{i, j_{u}} \widehat{\gamma}_{i}, \alpha_{d}=\zeta_{i, j_{d}} \widehat{\gamma}_{i}}$ where

$$
O^{d, r e l}\left(\alpha_{u}, \alpha_{d}\right)=1-\left(1-O\left(\alpha_{u}\right)\right)\left(1-O\left(\alpha_{d}\right)\right),
$$

in which $O\left(\alpha_{u}\right)$ and $O\left(\alpha_{d}\right)$ correspond to the outage probability functions of the uplink and downlink, respectively, obtained from (11). Also, $\zeta_{i, j_{u}}$ and $\zeta_{i, j_{d}}$ are obtained from (13) as $\zeta_{i, j_{u}}=p_{j_{u}} \mathbb{E}\left[\widehat{h}_{i, j_{u}}^{c r}\right] / p_{i}^{d r} \mathbb{E}\left[h_{i}^{d r}\right], \zeta_{i, j_{d}}=p_{j_{d}} \mathbb{E}\left[\widehat{h}_{i, j_{d}}^{c}\right] / p_{i}^{r d} \mathbb{E}\left[h_{i}^{r d}\right]$. Depending on the fading in the desired and interfering link for uplink and downlink, $O\left(\zeta_{i, j_{u}} \widehat{\gamma}_{i}\right)$ and $O\left(\zeta_{i, j_{d}} \widehat{\gamma}_{i}\right)$ in 20) can follow from any one of (11a), (11b), (11c), or (11d).

\section{B. Expressions of Link Capacity for D2D and cellular users}

Based on the expressions obtained for the outage probability of different links, we can now calculate the channel capacity for different users. For a given user having the SINR $\gamma$, the (ergodic) channel capacity is obtained according to the Shannon Theorem as follows:

$$
\begin{aligned}
\mathbb{E}\left[\log _{2}(1+\gamma)\right]=\int_{0}^{\infty} \log _{2}(1+\gamma) f_{\gamma}(\gamma) d \gamma & \\
= & \frac{1}{\ln 2} \int_{0}^{\infty} \frac{1-F_{\gamma}(\gamma)}{1+\gamma} d \gamma,
\end{aligned}
$$

where $f_{\gamma}(\gamma)$ and $F_{\gamma}(\gamma)$ are the probability density function (pdf) and cumulative density function (cdf) of the SINR, respectively. Therefore, the channel capacity of cellular users and D2D pairs are obtained as follows:

$$
\begin{aligned}
& R^{c}\left(\zeta_{j, i}\right)=f_{r}\left(O^{c}\left(\zeta_{j, i} \gamma\right)\right), \\
& R^{d, d i r}\left(\zeta_{i, j}\right)=f_{r}\left(O^{d, d i r}\left(\zeta_{i, j} \gamma\right)\right), \\
& R^{d, r e l}\left(\zeta_{i, j_{u}}, \zeta_{i, j_{d}}\right)=f_{r}\left(O^{d, r e l}\left(\zeta_{i, j_{u}} \gamma, \zeta_{i, j_{d}} \gamma\right)\right),
\end{aligned}
$$

where $f_{r}(g(\gamma))=\frac{1}{\ln (2)} \int_{0}^{\infty} \frac{1-g(\gamma)}{1+\gamma} d \gamma$, 22a obtains the channel capacity of cellular user $j$ sharing subchannel with D2D pair $i$, 22b) obtains the channel capacity of direct D2D pair $i$ reusing the subchannel of cellular user $j$, and 22c) obtains the channel capacity of relayed D2D pair $i$ reusing the subchannels of cellular users $j_{u}$ and $j_{d}$ in the uplink and downlink paths, respectively.

\section{EXPRESSIONS OF Frame DECODING ERror PROBABILITY}

Channel capacity is the largest achievable rate at which the information can be transmitted regardless of the decoding error probability, however, due to hardware limitations such as the modulation and demodulation techniques employed in the transmitter and receiver, there always exists a gap between the Shannon capacity and the achievable rate. Consider $n$ to to be the blocklength and $\varepsilon_{n}$ to be the corresponding decoding error probability. The maximum achievable bit-rate per symbol for quasi-static channels under finite block-length $(n<100)$ is tightly approximated by the following equation [18], [35].

$$
R^{*}\left(n, \varepsilon_{n}\right) \approx C-\sqrt{\frac{V}{n}} Q^{-1}\left(\varepsilon_{n}\right),
$$

where $C=\log _{2}(1+\gamma)$ is the channel capacity (in bits/second/Hz), $V=1-1 /(1+\gamma)^{2}$ is called the channel dispersion, $Q^{-1}$ is the inverse of Marcum Q-function $\left(Q(x)=\frac{1}{\sqrt{2 \pi}} \int_{x}^{\infty} e^{-\left(\frac{u^{2}}{2}\right)} d u\right)$. Assume that the transceiver modulation-demodulation mechanisms limit the achievable data rate to some coefficient of the channel capacity, i.e., $R^{*}(n, \varepsilon)=\xi \log _{2}(1+\gamma)$ where $\xi<1$ is constant. Then from 23) we have $C(1-\xi)=\sqrt{\frac{V}{n}} Q^{-1}\left(\varepsilon_{n}\right)$, and by inversing this equation, we have

$$
\begin{aligned}
& \varepsilon_{n}(\gamma)=Q\left((1-\xi) \sqrt{\frac{n}{V}} C\right) \\
& =Q\left((1-\xi) \sqrt{\frac{n}{1-\frac{1}{(1+\gamma)^{2}}}} \log _{2}(1+\gamma)\right)=Q(u(\gamma)),
\end{aligned}
$$

where $u(\gamma)=\frac{(1-\xi) \sqrt{n}}{\ln 2}\left(1-\frac{1}{(1+\gamma)^{2}}\right)^{-0.5} \ln (1+\gamma)$.

Theorem 1. For cellular user $j$ sharing a subchannel with D2D pair $i$ and utilizing frames of block-length $n$, the frame decoding error probability under quasi-static regime is obtained as

$$
\begin{array}{r}
\mathbb{E}\left\{\varepsilon_{n}(\gamma)\right\} \approx \sum_{i=1}^{L-1}\left(\omega_{i-1}-\omega_{i}\right) H\left(\zeta_{j, i}, \gamma_{i}\right)+\omega_{L} H\left(\zeta_{j, i}, \gamma_{L}\right) \\
\triangleq \bar{\varepsilon}_{n}\left(\zeta_{j, i}\right),
\end{array}
$$

$O_{L, L}(\alpha)=1-\left\{\frac{\left(K^{\prime}+1\right) e^{-K-K^{\prime}}}{\alpha(K+1)+\left(K^{\prime}+1\right)} \sum_{m=0}^{\infty} \frac{K^{m}}{m !}\left[\sum_{n=0}^{\infty} \frac{1}{n !}\left(\frac{\sqrt{K^{\prime}\left(K^{\prime}+1\right)}}{\alpha(K+1)+\left(K^{\prime}+1\right)}\right)^{2 n}{ }_{2} F_{1}\left(-n,-n, m+1 ; \frac{\alpha K}{2 K^{\prime}\left(K^{\prime}+1\right)}\right]\right\}\right.$, 
where $L>1$ is the integer approximation factor ${ }^{4} \omega_{i}=$ $\frac{0.5 / L}{\gamma_{i}-\gamma_{i-1}}, \gamma_{i}=\varepsilon_{n}^{-1}(0.5(1-i / L))$ for $0 \leq i \leq L-1$, and $\gamma_{L}=\varepsilon_{n}^{-1}(\Delta)$, in which $\Delta \ll 0.5 / L$ is an arbitrary small value constant, and

$$
H(\alpha, \gamma)= \begin{cases}H_{N}(\alpha, \gamma), & \text { for NLoS interference, } \\ H_{L}(\alpha, \gamma), & \text { for LoS interference, }\end{cases}
$$

where

$$
\begin{aligned}
& H_{N}(\alpha, \gamma)=\gamma-\frac{m^{\prime m^{\prime}}}{\Gamma\left(m^{\prime}\right)} \sum_{k=0}^{m-1} \frac{\Gamma\left(k+m^{\prime}\right)(m \alpha)^{k} \gamma^{k+1}}{(k+1) !\left(m^{\prime}\right)^{k+m^{\prime}}} \\
& \times{ }_{2} F_{1}\left(k+1, k+m^{\prime}, k+2,-\frac{m \alpha \gamma}{m^{\prime}}\right) \\
& H_{L}(\alpha, \gamma)=\frac{e^{-K}(m \alpha)^{m} \gamma^{m+1}}{\Gamma(m)} \times \\
& \sum_{j=0}^{\infty} \sum_{k=0}^{j} \frac{\Gamma(k+m) K^{j}{ }_{2} F_{1}\left(m+1, m+k, m+2, \frac{-m \alpha \gamma}{K+1}\right)}{j ! k !(K+1)^{m}(m+1)} .
\end{aligned}
$$

Proof. See Appendix C.

\section{Resource Allocation for UAV-Assisted D2D UNDERlaid CELlular NeTWORK}

Based on the performance metrics characterized in the previous sections, in this section, we formulate and solve the link-type selection (i.e., direct D2D or relayed D2D), subchannel and power allocation problem for a UAV-assisted D2D underlaid cellular network.

\section{A. Problem Formulation}

Let $\boldsymbol{\mu}^{\boldsymbol{r}} \in\{0,1\}^{M^{d}}$, where $\mu_{i}^{r}=1$ and $\mu_{i}^{r}=0$ denote that $i$ is relayed or direct D2D pair, respectively. Besides, let $\boldsymbol{\rho} \in\{0,1\}^{M^{d} \times M^{c}}$, where $\rho_{i, j}=0$ and $\rho_{i, j}=1$ show that $i \in \mathcal{M}^{d}$ reuses or does not reuse the subchannel of $j \in \mathcal{M}^{c}$, respectively. The link-type (direct or relayed), subchannel and power allocation optimization problem is formally stated as follows:

(P1) $\underset{\mathbf{p}^{d}, \mathbf{p}^{d r}, \mathbf{p}^{r d}, \mathbf{p}^{c}}{\operatorname{minimize}} \sum_{i}\left[\mu_{i}^{r}\left(p_{i}^{d r}+p_{i}^{r d}\right)+\left(1-\mu_{i}^{r}\right) p_{i}^{d}\right]+\sum_{j} p_{j}^{c}$

where $\mathbf{p}^{d}, \mathbf{p}^{d r}, \mathbf{p}^{r d}, \mathbf{p}^{c}$ belong to the set of all possible solutions of:

$$
\begin{gathered}
\underset{\boldsymbol{\rho}, \boldsymbol{\mu}^{r}, \mathbf{p}^{d}, \mathbf{p}^{d r}, \mathbf{p}^{r d}, \mathbf{p}^{c}}{\operatorname{maximize}} \sum_{i} \sum_{j}\left(1-\mu_{i}^{r}\right) \rho_{i, j} R^{d, d i r}\left(\zeta_{i, j}\right)+ \\
\quad \sum_{i} \sum_{j_{u}} \sum_{j_{d}} \mu_{i}^{r} \rho_{i, j_{u}} \rho_{i, j_{d}} R^{d, r e l}\left(\zeta_{i, j_{u}}, \zeta_{i, j_{d}}\right) \\
\text { s.t. } \rho_{i, j} \bar{\varepsilon}_{n}\left(\zeta_{j, i}\right) \leq p_{\varepsilon}, \quad \forall i, j, \\
\quad R^{c}\left(\zeta_{j, i}\right) \geq \rho_{i, j} \widehat{R}^{c}, \quad \forall i, j, \\
\quad \mu_{i}^{r} \in\{0,1\}, \rho_{i, j} \in\{0,1\}, \quad \forall i, j, \\
\quad\left(1-\mu_{i}^{r}\right) \sum_{j} \rho_{i, j} \leq 1, \quad \forall i
\end{gathered}
$$

\footnotetext{
${ }^{4}$ We will show through numerical results that assigning $L=4$ which is equivalent to a 4-level piece-wise linearization of [24], tightly fits the corresponding exact expression with negligible error.
}

$$
\begin{aligned}
& 2\left(1-\mu_{i}^{r}\right)+\mu_{i}^{r} \sum_{j} \rho_{i, j}=2, \quad \forall i \\
& \sum_{i} \rho_{i, j} \leq 1, \quad \forall j, \\
& \left(p_{i}^{d}, p_{i}^{d r}, p_{i}^{r d}, p_{j}^{c}\right) \leq\left(\bar{p}_{i}^{d}, \bar{p}_{i}^{d r}, \bar{p}_{i}^{r d}, \bar{p}_{j}^{c}\right), \quad \forall i, j, \\
& \left(\rho_{i, j} \underline{p}_{i}^{d}, \rho_{i, j} \underline{p}_{i, j}^{d r}, \rho_{i, j} \underline{p}_{i, j}^{r d}, \rho_{i, j} \underline{p}_{j, i}^{c}\right) \leq\left(p_{i}^{d}, p_{i}^{d r}, p_{i}^{r d}, p_{j}^{c}\right), \\
& \forall i, j .
\end{aligned}
$$

From (30), the objective of the inner problem is to first maximize the aggregate achievable data rate of all D2D pairs subject to the power constraints of the all users and QoS (ergodic rate and reliability) constraints of cellular users. Also, (30a) calculates the objective to be maximized as the total channel capacity of all relayed and direct D2D pairs. (30b) and (30c) guarantee that the average decoding error probability and channel capacity of each cellular user $j$ are kept below the maximum allowed error probability $p_{\varepsilon}$ and beyond the minimum allowed channel capacity $\widehat{R}^{c}$, respectively. 30c can be also viewed as the latency constraint where $T_{j, i}=$ $n_{0} / R^{c}\left(\zeta_{j, i}\right)$ is the latency of cellular user $j$ if its channel is reused by D2D pair $i$ and $n_{0}$ is the total amount of data to be transferred, and similarly, $\widehat{T}=n_{0} / \widehat{R}$ is the maximum acceptable latency of each cellular user. The constraints (30e), (30f), and (30g) ensure that inactive direct D2D pairs are allocated to no subchannel, and each active direct D2D pair reuses the subchannel of one cellular user, and each active relayed D2D pair reuses the subchannels of two cellular users, respectively, and finally 30h and 30i guarantee that the power constraints of all D2D pairs and cellular users hold. Note that, 30i] guarantees that the interference-limited assumption always hold.

To solve the considered MINLP problem, our methodology is as follows:

1) For a given subchannel assignment, we first rewrite P1 as shown in $\mathbf{P 2}$ of Section V.B to optimize power allocations. We then solve the inner problem of $\mathbf{P 2}$ to get closed-form optimal power allocations by reformulating the power variables in terms of new variable $\eta=p_{i} / p_{j}$. The optimal value of $\eta$ (denoted by $\eta^{*}$ ) corresponds to all optimal points $\left(p_{i}, p_{j}\right)$ lying in the line $p_{i}=\eta^{*} p_{j}$ (provided that the feasibility and interference limited assumptions hold, which is verified in the proof of Theorem 2).

2) Then, from the space of optimal powers obtained from the inner problem of P2, we derive a power allocation solution corresponding to the minimum aggregate transmit power of the UAVs and all D2D and cellular devices, as in the outer problem of $\mathbf{P 2}$.

3) Then, we formulate our problem as one-to-many matching problem which is solved by first obtaining the optimal subchannel and power allocation of the maximum weighted one-to-one matching problem (assuming no UAV relays) and then we extend the scheduling scheme to one-to-many matching problem with UAV relays (Section V.C).

Our solution approach is different from iterative alternating optimization methods where a problem is typically split into several sub-problems and each sub-problem solves only one 


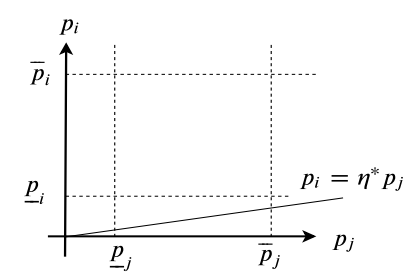

a) $\eta^{*}<\underline{p}_{i} / \bar{p}_{j}$

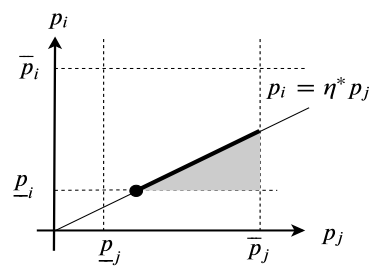

b) $\underline{p}_{i} / \bar{p}_{j} \leq \eta^{*}<\underline{p}_{i} / \underline{p}_{j}$

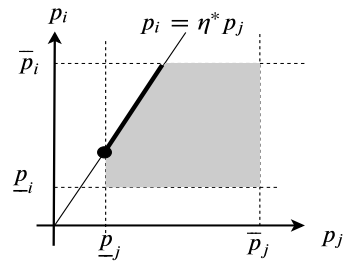

c) $\underline{p}_{i} / \underline{p}_{j}<\eta^{*} \leq \bar{p}_{i} / \underline{p}_{j}$

- The unique point corresponding to the solution of the outer problem

The line corresponding to the solution space of the inner problem

Feasible region

Fig. 2. Illustration of three possible cases of the solution of inner and outer problems. The thick lines show the solution space of the inner problem, and the small filled circles show the single point corresponding to the solution of the outer problem.

optimization variable given the remaining optimization variables from the previous iteration.

\section{B. Optimal Power Allocations for D2D and cellular users}

In the following, we present the closed-form power allocations for any possible subchannel assignment. Suppose that D2D pair $i \in \mathcal{M}^{d}$ reuses the subchannel allocated to cellular user $j \in \mathcal{M}^{c}$. Let $p_{j} \triangleq p_{j}^{c}$ be the transmit power of cellular user $j$. Without loss of generality, we assume that $i$ is a direct D2D pair with transmit power $p_{i} \triangleq p_{i}^{d}$, however one can easily verify that the following analysis is also valid for the uplink of relayed D2D pair $i$ with transmit power $p_{i} \triangleq p_{i}^{d r}$, and for the downlink of relayed D2D pair $i$ with transmit power $p_{i} \triangleq p_{i}^{r d}$. The power allocation for D2D pair $i$ and cellular user $j$ corresponding to 29) and 30 is stated as follows:

$$
\underset{p_{i}, p_{j}}{\operatorname{minimize}} p_{i}+p_{j}
$$

where $p_{i}, p_{j}$ belong to the set of all possible solutions of:

$$
\begin{aligned}
\underset{p_{i}, p_{j}}{\operatorname{maximize}} & R^{d, d i r}\left(\zeta_{i, j}\right) \\
\text { s. t. } & R^{c}\left(\zeta_{j, i}\right) \geq \widehat{R}^{c} \\
& \bar{\varepsilon}_{n}\left(\zeta_{j, i}\right) \leq p_{\varepsilon}, \\
& \left(\underline{p}_{i}, \underline{p}_{j}\right) \leq\left(p_{i}, p_{j}\right) \leq\left(\bar{p}_{i}, \bar{p}_{j}\right),
\end{aligned}
$$

where $\underline{p}_{i} \triangleq \underline{p}_{i, j}$ and $\underline{p}_{j} \triangleq \underline{p}_{j, i}$ are obtained from 10 and $\bar{\varepsilon}_{n}($.$) is obtained from 25$.

Theorem 2. The solution to power allocation sub-problem given in (31) and (32) is:

$$
\left(p_{i}^{*}, p_{j}^{*}\right)= \begin{cases}\left(\eta^{*} \underline{p}_{j}, \underline{p}_{j}\right), & \text { if } \underline{p}_{i} / \underline{p}_{j} \leq \eta^{*} \\ \left(\underline{p}_{i}, \underline{p}_{i} / \eta^{*}\right), & \text { if } \underline{p}_{i} / \bar{p}_{j} \leq \eta^{*}<\underline{p}_{i} / \underline{p}_{j}, \\ \emptyset, & \text { if } \eta^{*}<\underline{p}_{i} / \bar{p}_{j},\end{cases}
$$

where $\eta^{*}=\min \left\{\eta_{1}^{*}, \eta_{2}^{*}, \bar{p}_{i} / \underline{p}_{j}\right\}$ in which $\eta_{1}^{*}$ and $\eta_{2}^{*}$ are unique solution values of $\eta$ in the equality constraints $R^{c}\left(\eta \mathbb{E}\left[\widehat{h}_{j, i}\right] / \mathbb{E}\left[h_{j}\right]\right)=\widehat{R}^{c}$ and $\bar{\varepsilon}_{n}\left(\eta \mathbb{E}\left[\widehat{h}_{j, i}\right] / \mathbb{E}\left[h_{j}\right]\right)=p_{\varepsilon}$, respectively.

Proof. We first find the solution to the inner optimization subproblem (32). By considering $\eta=p_{i} / p_{j}$ as the optimization variable, 32 can be rewritten as

$$
\underset{\eta}{\operatorname{maximize}} R^{d, d i r}\left(k_{1} / \eta\right)
$$

$$
\begin{array}{ll}
\text { s. t. } & R^{c}\left(k_{2} \eta\right) \geq \widehat{R}^{c}, \\
& \bar{\varepsilon}_{n}\left(k_{2} \eta\right) \leq p_{\varepsilon}, \\
& \underline{p}_{i} / \bar{p}_{j} \leq \eta \leq \bar{p}_{i} / \underline{p}_{j},
\end{array}
$$

where $k_{1}=\mathbb{E}\left[\widehat{h}_{i, j}\right] / \mathbb{E}\left[h_{i}\right]$, and $k_{2}=\mathbb{E}\left[\widehat{h}_{j, i}\right] / \mathbb{E}\left[h_{j}\right]$. First, we prove that $\bar{\varepsilon}\left(k_{2} \eta\right)$ in $34 \mathrm{c}$ ) is an increasing function of $\eta$. From (25) and (39), we have

$$
\begin{aligned}
& \bar{\varepsilon}_{n}\left(k_{2} \eta\right)=\sum_{i=1}^{L-1}\left(\omega_{i-1}-\omega_{i}\right) H\left(k_{2} \eta, \gamma_{i}\right)+\omega_{L} H\left(k_{2} \eta, \gamma_{L}\right) \\
& =\sum_{i=1}^{L-1}\left(\omega_{i-1}-\omega_{i}\right) \int_{0}^{\gamma_{i}} O^{c}\left(k_{2} \eta \gamma\right) d \gamma+\omega_{L} \int_{0}^{\gamma_{L}} O^{c}\left(k_{2} \eta \gamma\right) d \gamma .
\end{aligned}
$$

Noting that $\omega_{i-1}>\omega_{i}, \forall i$, and also by noticing that $O^{c}\left(k_{2} \eta \gamma\right)=\operatorname{Pr}\left\{\frac{y}{x} \leq k_{2} \eta \gamma\right\}$ is an increasing function of $\eta$ (where $y$ and $x$ are fading variables associated with the channel of cellular user $j$ and the interfering channel from user $i$ ), it is clear that $\bar{\varepsilon}_{n}\left(k_{2} \eta\right)$ is an increasing function of $\eta$. Hence, we can equivalently replace constraint 34c by $\eta \leq \eta_{2}^{*}$. Now we prove that $R^{c}\left(k_{2} / \eta\right)$ is also a monotonically increasing function of $\eta$. From 22a and the definition of $f_{r}($.$) , we have$

$$
\begin{aligned}
& R^{c}\left(k_{2} \eta\right)=\frac{1}{\ln (2)} \int_{0}^{\infty} \frac{1-O^{c}\left(k_{2} \gamma \eta\right)}{1+\gamma} d \gamma= \\
& \quad \frac{1}{\ln (2)} \int_{0}^{\infty} \frac{1-O^{c}(\gamma)}{k_{2} \eta+\gamma} d \gamma
\end{aligned}
$$

Noting the fact that $O^{c}(\gamma) \leq 1$, from 35 we conclude that $R^{c}\left(k_{2} \eta\right)$ is an decreasing function of $\eta$ and so, (34b) can be replaced by $\eta \leq \eta_{1}^{*}$. In a similar way, we could verify that $R^{d, \operatorname{dir}}\left(k_{1} / \eta\right)$ is an increasing function of $\eta$. Thus, we can rewrite (34) as

$$
\begin{aligned}
\underset{\eta}{\operatorname{maximize}} & R^{d, d i r}\left(k_{1} / \eta\right) \\
& \underline{p}_{i} / \bar{p}_{j} \leq \eta \leq \min \left\{\eta_{1}^{*}, \eta_{2}^{*}, \bar{p}_{i} / \underline{p}_{j}\right\},
\end{aligned}
$$

and hence we conclude that $\eta^{*}=\min \left\{\eta_{1}^{*}, \eta_{2}^{*}, \bar{p}_{i} / \underline{p}_{j}\right\}$ is the unique solution to 34 provided that $\eta^{*} \geq \underline{p}_{i} / \bar{p}_{j}$; otherwise the problem is infeasible. Fig. 2 shows that depending on $\eta^{*}$ (where $0<\eta^{*} \leq \bar{p}_{i} / \underline{p}_{j}$ ), three possible cases may happen. For case (a) where $\eta^{*}<\underline{p}_{i} / \bar{p}_{j}$, there exists no feasible point. The set of optimal solutions of the inner problem for cases (b) and (c) are illustrated as the thick lines corresponding to the intersection of the feasible region (displayed in gray) and the 


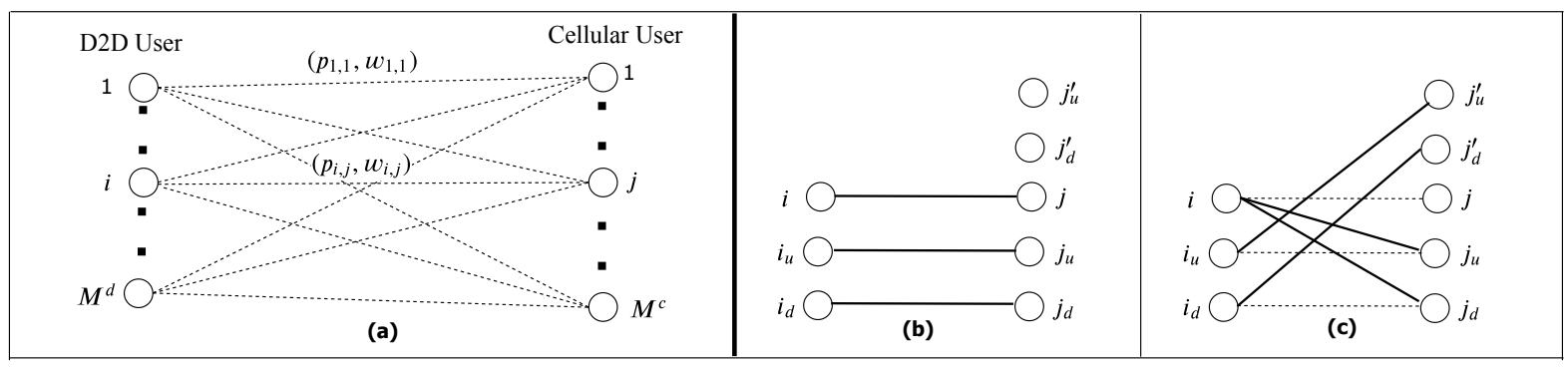

Fig. 3. Bipartite graph for the matching problem. Fig. (a) shows the one-to-one matching for Algorithm 1 for the case when no UAV relay is used. Figs (b) and (c) show the procedure of changing a direct D2D link to a relayed link in Algorithm 2.

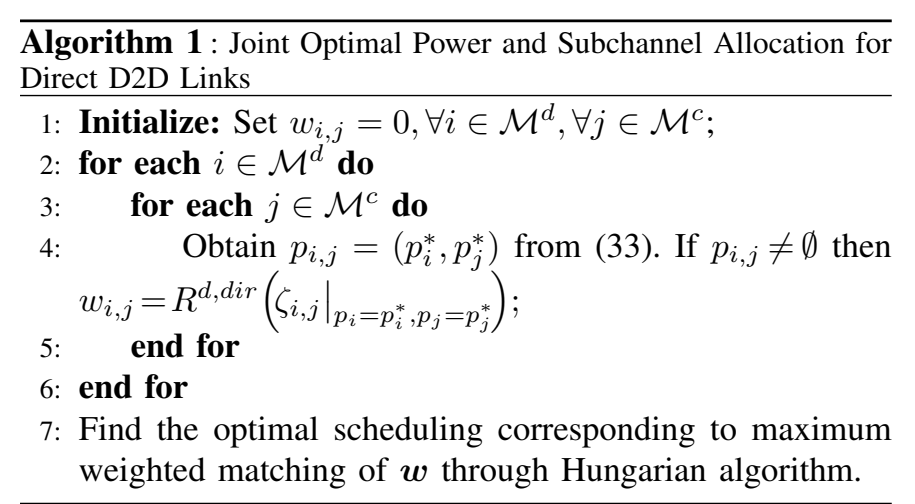

line $p_{i}=\eta^{*} p_{j}$. Now, referring to problem $\sqrt{31}$ and constraint (32d), the optimal power allocation is obtained as 33, which is shown in black filled circles in the figure.

\section{Link-type, Subchannel, and Power Allocation for D2D Pairs}

In order to solve the link-type, subchannel and power allocation problem stated in (29) and (30), first we consider the case where no UAV relay is involved and all D2D pairs can only establish direct links. As seen in Fig. 3 -a, consider a bipartite graph composed of two sets of vertices, namely D2D pairs and cellular users whose subchannels are going to be reused by D2D pairs. Each D2D pair can only reuse the subchannel of one cellular user and each cellular user can share its subchannel to only one D2D pair, thus the scheduling is a one-to-one maximum weighted matching problem which can optimally be solved by Hungarian algorithm. The procedure of calculating the powers and weights for each link and obtaining the optimal solution is described in Algorithm 1.

In order to further increase the throughput of D2D links, we then update Algorithm 1 by replacing direct D2D links with poor QoS with relayed links. This problem is modeled as a one-to-many matching game, where each D2D pair may reuse the subchannel of one cellular user (if it establishes a direct D2D link) or the subchannels of two cellular users (for the case of relayed D2D links), but each cellular user still shares its subchannel to at most one D2D pair. Let $i=\rho^{d}(j)$ denotes a D2D pair $i$ reusing the subchannel of cellular user $j$, $j=\rho^{c}(i)$ denotes a cellular user $j$ whose subchannel is reused by D2D pair $i$ in the direct link mode, and $\left(j_{u}, j_{d}\right)=\rho^{c}(i)$ denotes cellular users $j_{u}$ and $j_{d}$ whose subchannels are reused respectively in the uplink and downlink paths of the relayed link of D2D pair $i$. The relay-assisted scheduling and power allocation problem is proposed in Algorithm 2.

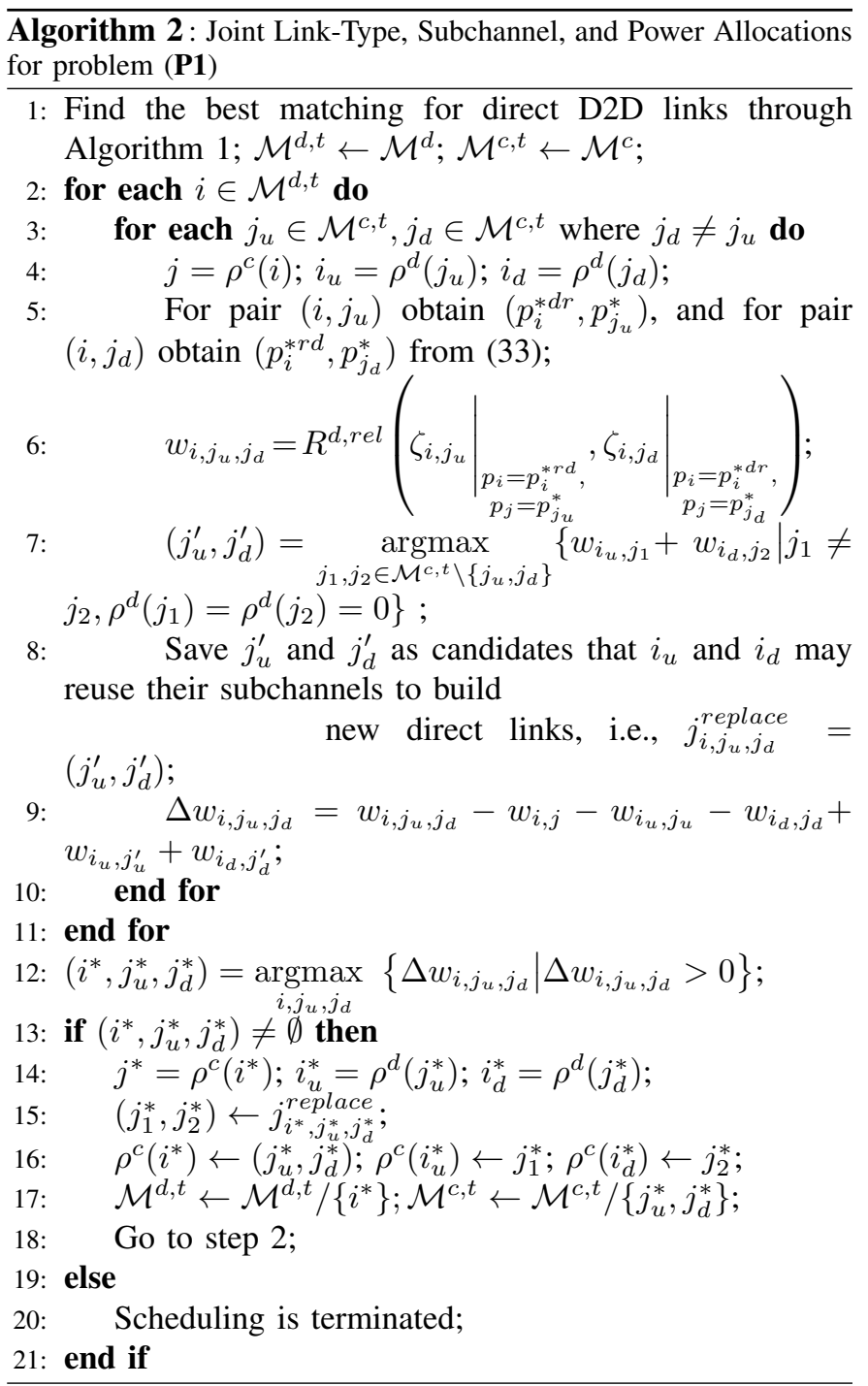

As seen in Algorithm 2, first we find the optimal scheduling for direct D2D links establishment through Hungarian algorithm, and then we form the set of temporary candidates of relayed D2D pairs as $\mathcal{M}^{d, t}$ and cellular users whose subchannels can be reused by relayed D2D candidates as $\mathcal{M}^{c, t}$. In order to see how relayed links are established, from Fig. 3-b, suppose $j, j_{u}, j_{d} \in \mathcal{M}^{d, t}$ have initially shared their subchannels to direct D2D pairs $i, i_{u}, i_{d}$ respectively. We 
denote $i_{u}=\emptyset$, and $i_{d}=\emptyset$ for the case when $j_{u}$ or $j_{d}$ have shared their subchannels to none of D2D links. In Steps 2-13, we search among all possible allocations of $j_{u}, j_{d} \in \mathcal{M}^{c, t}$ to establish a relayed connection to some $i \in \mathcal{M}^{d, t}$ which results in the maximum throughput increase $\Delta w_{i, j_{u}, j_{d}}$. In assigning the subchannels of $j_{u}$ and $j_{d}$ to D2D pair $i$, as seen in Step 10, and Fig. 3-c, the terms $w_{i, j}, w_{i_{u}, j_{u}}$, and $w_{i_{d}, j_{d}}$ are deducted from $\Delta w_{i, j_{u}, j_{d}}$ since their corresponding connections are lost, and instead, the terms $w_{i, j_{u}, j_{d}}, w_{i_{u}, j_{u}^{\prime}}$, and $w_{i_{d}, j_{d}^{\prime}}$ are added. Note that, in order to replace the direct links of $i_{u}$ (if $i_{u} \neq \emptyset$ ) and $i_{d}$ (if $i_{d} \neq \emptyset$ ) from $j_{u}$ and $j_{d}$, to $j_{u}^{\prime}$ and $j_{d}^{\prime}$, as seen in Step 8, we search through all possible candidates that may maximize the sum of the weights of direct links $w_{i_{u}, j_{u}^{\prime}}+w_{i_{d}, j_{d}^{\prime}}$. After finding the best candidate $\left(i^{*}, j_{u}^{*}, j_{d}^{*}\right)$ in Step 14, we set all required allocations in Steps 16-18, and remove $\left\{i^{*}\right\}$ and $\left\{j_{u}^{*}, j_{d}^{*}\right\}$ from the candidate sets $\mathcal{M}^{d, t}$ and $\mathcal{M}^{c, t}$, respectively in Step 19, and then we continue the procedure again until no allocation can be found that improves $\Delta w$.

As a benchmark for the proposed Algorithm 2, we consider two versions of low complexity greedy algorithms. In the first algorithm denoted by Greedyl, we first obtain the optimal subchannel and power solution through Algorithm 1 and then D2D pairs sequentially change their direct links to relayed links (in a greedy manner by selecting uplink and downlink channels resulting in the highest throughput) provided that the corresponding user's throughput is increased by changing its link-type. In the second version denoted by Greedy2, after obtaining the optimal solution of Algorithm 1, we first sort D2D pairs in ascending order according to the achieved directlink data rates, aiming at D2D pairs with low data rates to opt for higher quality relayed links, and then a similar mechanism as Greedyl is employed.

\section{Complexity Analysis}

For one-to-one matching game, described in Algorithm 1, the standard Hungarian algorithm can be solved with timecomplexity of $O\left(\left(M^{d}\right)^{2} M^{c}\right)$ [36]. Regarding the complexity of Algorithm 2, in order to make the analysis simpler, we calculate the worst case for the upper-bound time-complexity. The steps 5-10 are executed by $O(1)$. More specifically, we show this for Step 8 (which is potentially of most complexity among others). After finishing the initialization step and before starting Step 2, for each $i$ we can create the vector of elements $w_{i}^{\text {sorted }}(j)$ by sorting $w_{i, j}$ for all $j$ such that $\rho(j)=0$, and thus, by doing this for all $i$, we can create the sorted weight matrix $w^{\text {sorted }}$ with an overall complexity of $O\left(M^{c} M^{d}\right)$. This way, Step 8 can always be executed by $O(1)$; Hence the overall complexity of each iteration for Steps 2-13 in the worst case (by ignoring the user removals from $\mathcal{M}^{c, t}$, i.e., by assuming $\left.\left|\mathcal{M}^{c, t}\right|=M^{c}\right)$ is $O\left(M^{d}\left(M^{c}\right)^{2}\right)$. Now, since the procedure is run again, in Step 20, after the removal of $i^{*}$ from $\mathcal{M}^{d, t}$ in Step 19, the complexity is obtained as $O\left(\left(M^{d} M^{c}\right)^{2}\right)$. Thus, by considering the execution of Hungarian Algorithm in Step 1 , the overall upper-bound complexity will be $O\left(\left(M^{d} M^{c}\right)^{2}\right)+$ $O\left(\left(M^{d}\right)^{2} M^{c}\right)=O\left(\left(M^{d} M^{c}\right)^{2}\right)$. Similar to the discussion presented above, the complexity of Greedyl Algorithm is easily obtained as $O\left(\left(M^{d}\right)^{2} M^{c}\right)+O\left(M^{d}\left(M^{c}\right)^{2}\right)$. Complexity of Greedy2 Algorithm is similar to that of Greedy1 added by the complexity of sorting D2D rates; thus it is found to be $O\left(M^{d} \log \left(M^{d}\right)\right)+O\left(\left(M^{d}\right)^{2} M^{c}\right)+O\left(M^{d}\left(M^{c}\right)^{2}\right)=$ $O\left(\left(M^{d}\right)^{2} M^{c}\right)+O\left(M^{d}\left(M^{c}\right)^{2}\right)$.

\section{Simulation Results}

Consider a cell where the BS is located at the center and several cellular and D2D users are randomly located within the cell. Simulation parameters are listed in Table II] The network frequency and path-loss parameter values are considered according to the experimental results given in [37]. In all scenarios, except for Fig. 9 in Section VI-4 (wherein simulation measures are obtained versus fixed values of D2D pairs distances), cellular users as well as D2D transmitters and receivers are randomly located within the cell area. Based on the positions of the users and UAVs, the probability of LoS relating to main and interference signals of aerial and terrestrial links are obtained for each user according to (4) and (5) respectively. By default, we consider two UAVs located at $(450,0)$ and $(-450,0)$ with respect to the center, and the height of UAVs are considered to be $300 \mathrm{~m}$. In order to compensate for the severe path-loss in mm-wave frequencies, it is needed to employ beamforming in the antennas of transmitters and receivers. For each transmitting antenna, we consider that the beamforming is applied in a way that the maximum directivity of the antenna is steered toward the desired link. Therefore, the main receiver receives the desired signal with maximum directivity gain $A^{\max }$, and the interfered receiver is subject to a directivity gain lower than $A^{\max }$. Let $\theta$ be the angle between a desired link and its corresponding interfering link. We model the antenna beamforming directivity pattern similar to the Gaussian-like shape proposed and employed in [38] and [39] as $A(\theta)=\max \left\{A^{\max } \exp \left(-0.69 \frac{\mathcal{N}(\theta)^{2}}{\theta_{3 \mathrm{~dB}}^{2}}\right), A^{\text {min }}\right\}$ where $\mathcal{N}(\theta)=\bmod _{2 \pi}(\theta+\pi)-\pi, A^{\max }$ is the maximum gain (which is radiated toward the main receiver), $A^{\text {min }}$ is a minimum constant, and $\theta_{3 \mathrm{~dB}}$ is the half-power beamwidth (i.e., $\left.A\left(\theta_{3 \mathrm{~dB}}\right)=A^{\max } / 2\right)$. In what follows, simulation parameter values are taken from Table II] unless explicitly stated otherwise. The results for all following figures are obtained by Monte-Carlo.

1) Validation of derived expressions: In order to validate the derived expressions, Fig. 4 depicts the outage probability expressions of $O_{L, N}$ in (15), and $O_{N, N}$ in (17) for different values of fading shape factors of $m$ and $K$. It is seen that the values obtained through derived expressions (solid lines), exactly match the values obtained through Monte-Carlo simulation by generating corresponding Rician and Nakagami$\mathrm{m}$ random values and taking the average of the outage. Similar procedure have been used in order to validate the estimated frame decoding error probability expressions obtained in 26a and (26b) as seen in Fig. 5. By considering 4-level piece-wise linearization $(L=4)$, it is seen that both derived expressions of 26a (first figure) and 26b (second figure) tightly match the exact values.

2) Performance versus the height of UAV: Fig. 6 shows how increasing the height of UAV and also the number of cellular users influence the performance of the system by 
TABLE II

SIMULATION PARAMETERS

\begin{tabular}{|l|l|l|l|}
\hline Parameter & Description & Parameter & Description \\
\hline Frequency & $28 \mathrm{GHz}$ & Noise power & $1.1 \times 10^{-12}$ Watts \\
Average path-loss for NLoS links & $72+29.2 \log _{10}(d) \mathrm{dB}$ & $\left(M^{c}, M^{d}\right)$ & $(18,10)$ \\
Average path-loss for LoS links & $61.4+20 \log _{10}(d) \mathrm{dB}$ & Cell area & $1600 \times 1600 \mathrm{~m}^{2}$ \\
$\left(\bar{p}_{i}^{d}, \bar{p}_{i}^{d r}, \bar{p}_{i}^{r d}, \bar{p}_{j}^{c}\right)$ & $(0.1,0.1,1,0.1)$ watts & $\widehat{R}^{c}$ & $8 \mathrm{bps} / \mathrm{Hz}$ \\
$(B, C)$ in $[4][31]$ & $(0.1396,11.95)$ & Fading factors $(m, K)$ & $(2,12 \mathrm{~dB})$ \\
UAV height, BS height & $250 \mathrm{~m}, 50 \mathrm{~m}$ & $\left(A^{\text {max }}, \theta_{3 \mathrm{~dB}}\right)$ & $\left(25 \mathrm{~dB}, 15^{\circ}\right)$ \\
$\left(p_{\varepsilon}, k_{n}\right)$ & $\left(10^{-4}, 5\right)$ & & \\
\hline
\end{tabular}
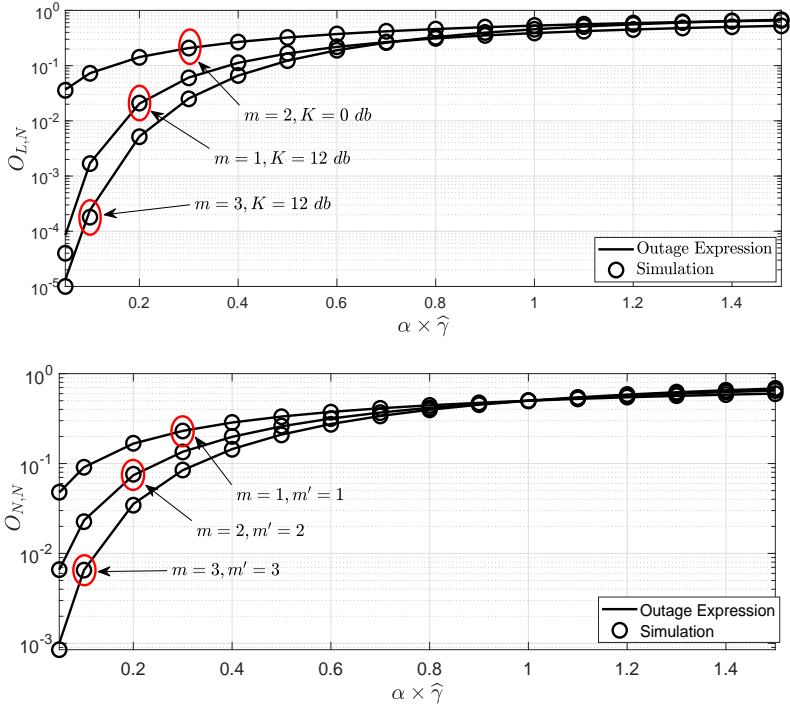

Fig. 4. Comparison of outage probability of derived expressions in 15 (first figure) and (17) (second figure) and their corresponding values obtained through simulation.
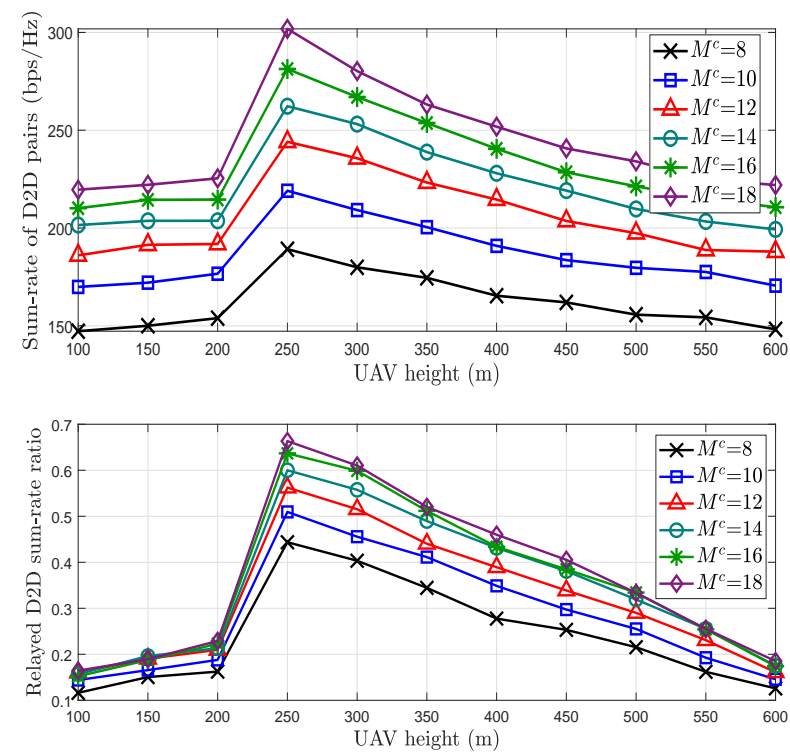

Fig. 6. Comparison of the sum-rate and relayed D2D sum-rate ratio of D2D pairs versus the height of UAV and number of available cellular users.
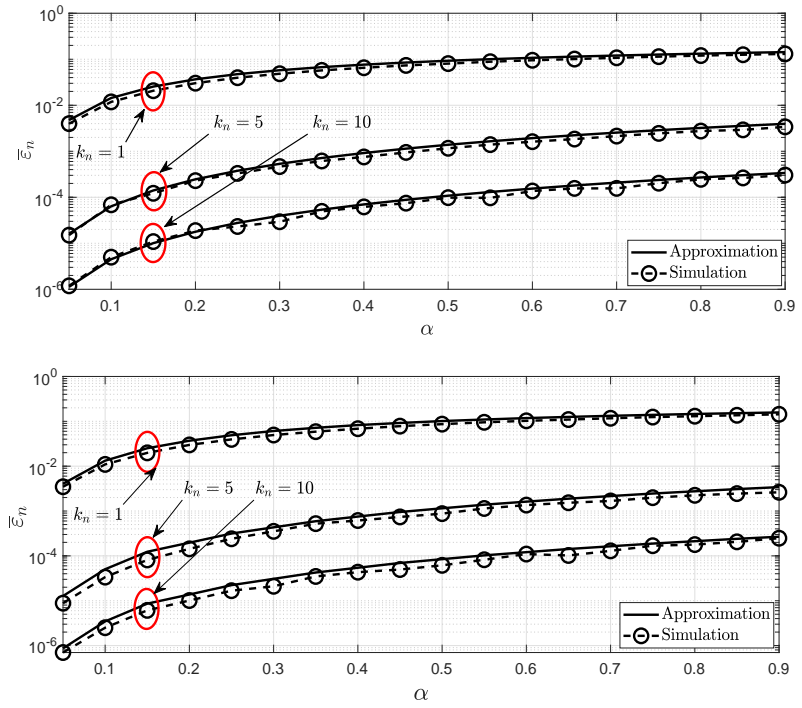

Fig. 5. Comparison of the frame decoding error probability of 26a) (first figure) and 26b (second figure) and corresponding exact values obtained from simulations.
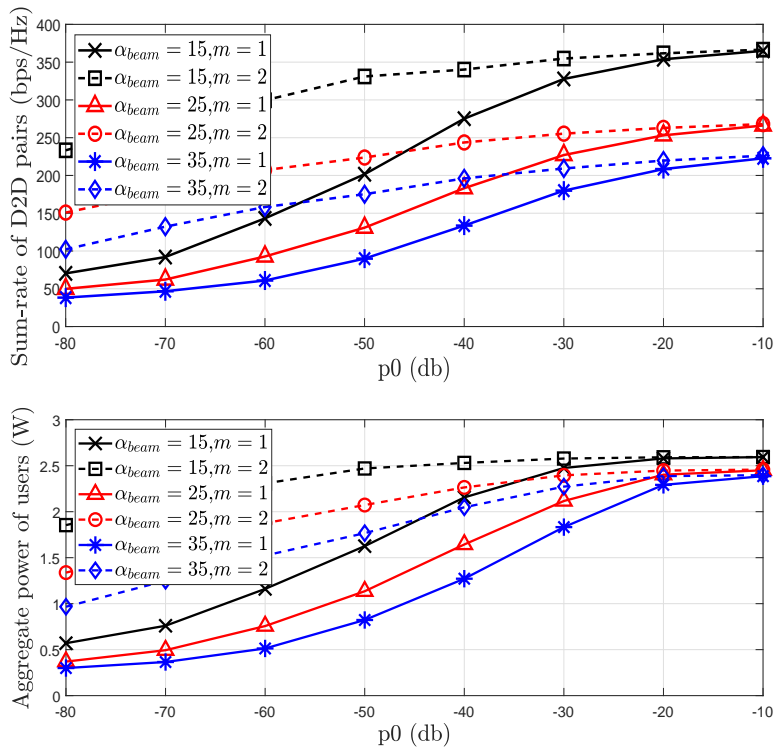

Fig. 7. Comparison of the sum-rate of D2D pairs, and aggregate power of users versus the decoding error probability $\left(p_{\varepsilon}\right)$, and $\theta_{3 \mathrm{~dB}}$ and fading shaping factor $m$. 

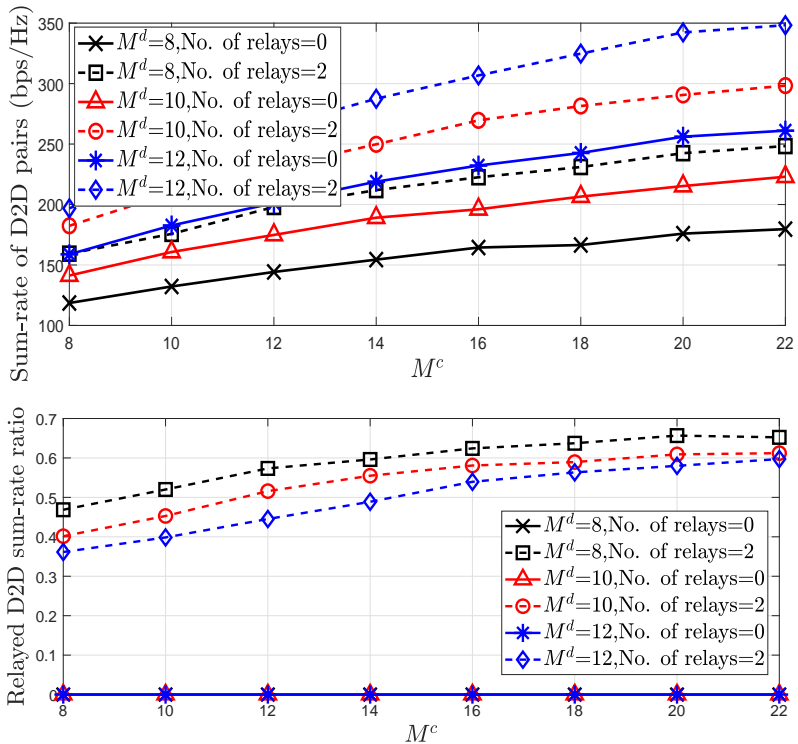

Fig. 8. Sum-rate of D2D pairs and relayed D2D sum-rate ratio for the cases when UAV is or is not employed versus number of cellular users and D2D pairs.

using Algorithm 2. We note that an increase in the number of cellular users provide more pairing options for D2D users which always leads to an increase in the system throughput. Also, we note that there is an optimal height (around $250 \mathrm{~m}$ ) beyond which the path loss due to high link distance, and below which the NLoS probability are the dominating factors of the performance degradation. For example, while it can be seen that for $M^{c}=16$, the relayed $\mathrm{D} 2 \mathrm{D}$ sum-rate ratio (the ratio of the sum-rate of relayed D2D pairs to that of all D2D pairs) is 0.16 and 0.17 for UAV height of $100 \mathrm{~m}$ and $600 \mathrm{~m}$ respectively, it is near 0.64 for the height of $250 \mathrm{~m}$ corresponding to the optimal total sum-rate of $280 \mathrm{bps} / \mathrm{Hz}$ for D2D pairs. This figure also shows the significance of UAV relays compared to terrestrial relays.

3) Performance versus decoding error probability : Fig. 7 shows performance of Algorithm 2 versus maximum acceptable frame decoding error probability of cellular users (i.e., $p_{\varepsilon}$ ) for the half-power angle $\theta_{3 \mathrm{~dB}}$ values of $15^{\circ}, 25^{\circ}$, and $35^{\circ}$ and fading shape factor of $m=1$ and $m=2$. Firstly, it is seen how increasing $p_{\varepsilon}$ of cellular users increases the sum-rate of D2D pairs for different values of $m$ and $\theta_{3 \mathrm{~dB}}$. This is because allowing cellular users to tolerate more frame decoding error probability will permit D2D users to impose more interference on their underlaid cellular users. Secondly, it seen how increasing the fading shape factor $m$ (which corresponds to less fading variance) results in the increase of D2D sum-rate. The increase is more evident for lower values of $p_{\varepsilon}$ since for lower values of $p_{\varepsilon}$ more stringent power control is employed for D2D users with severe fading conditions of $m=1$. Finally, increasing the half-power beamwidth results in decreasing the D2D sum-rate due to more interference imposed on cellular users.

4) UAV-assisted D2D vs Direct D2D: Fig. 8 illustrates the sum-rate of D2D users, aggregate transmit power of users,
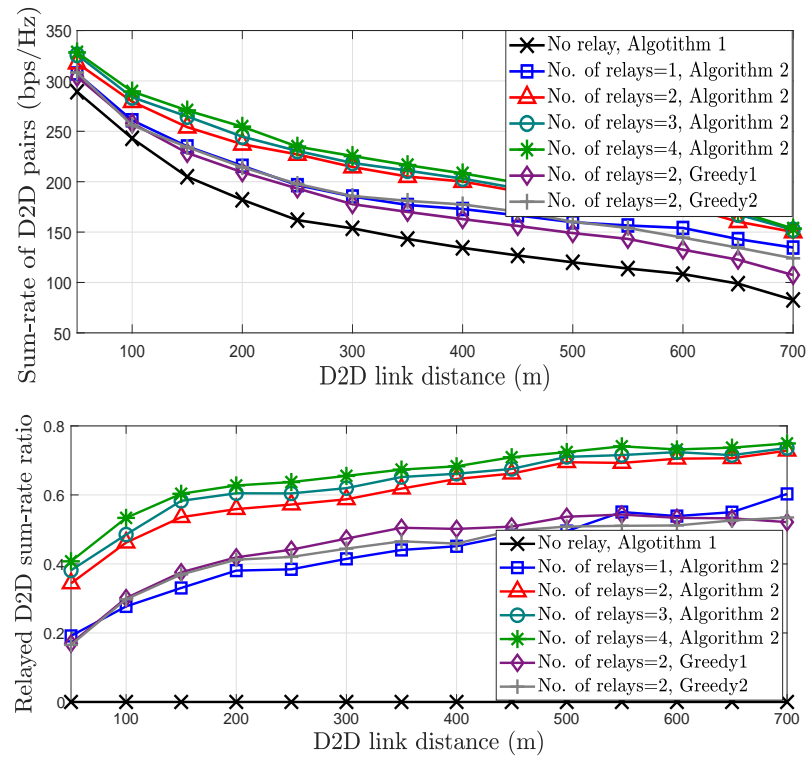

Fig. 9. Sum-rate of D2D pairs, aggregate power of users and relayed D2D sum-rate ratio versus the distance between D2D receiver and transmitter, and number of UAV relays.

and relayed D2D sum-rate ratio versus the number of cellular users and number of D2D pairs computed through Algorithm 1 (with no relays) and Algorithm 2 (with UAV relays). It is seen how increasing $M^{c}$ and $M^{d}$ and also employing UAV relays can increase the aggregate throughput of D2D pairs. For example, for $M^{c}=14$ and $M^{d}=8$, Algorithm 2 enhances the D2D aggregate throughput from 154 to $210 \mathrm{bps} / \mathrm{Hz}$ which is about $36 \%$ enhancement. Also, increasing $M^{d}$ from 8 to 12 , increases the throughput from 154 to 219 with no relay, and from 210 to 285 with UAV relays.

To illustrate the significance of UAVs on the performance of D2D pairs with different link distances, we consider a maximum of 4 UAVs located at $( \pm 450,0)$ and $(0, \pm 450)$. The aggregate D2D throughput and relayed D2D sum-rate ratio versus the number of UAVs and D2D links' distance is shown in Fig. 9. We have shown the performance of Algorithm 1 when no UAV exists, Algorithm 2 for 1-4 number of UAVs, and that of the proposed greedy algorithms when two UAVs are employed. As can be seen, Algorithm 2 outperforms the greedy algorithm to a great extent. We can also see that the increase in D2D link distance will generally reduce the aggregate throughput of D2D pairs, however, the higher link distance, the more UAVs assist to the sum-rate of D2D pairs. For example, it is seen that for the link distance of $200 \mathrm{~m}$, Algorithm 1 (with no UAV relay) obtains the sum-rate of 182 bps/Hz, while Algorithm 2 with 1 and 2 UAVs obtains the sum-rate of 214 and $237 \mathrm{bps} / \mathrm{Hz}$ which correspond to $17 \%$ and $30 \%$ enhancement, respectively. However, for D2D link distance of $600 \mathrm{~m}$, Algorithm 1 (with no relay) obtains the sum-rate of $108 \mathrm{bps} / \mathrm{Hz}$, while Algorithm 2 with 1 and 2 UAVs obtains the sum-rate of 154 and $176 \mathrm{bps} / \mathrm{Hz}$ which correspond to $43 \%$ and $63 \%$ enhancement respectively. Besides, it is seen that employing more than two UAVs will not have significant impact on the performance. 


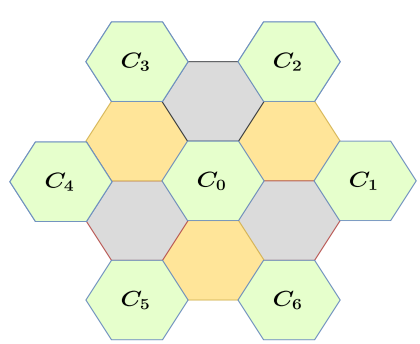

(a)

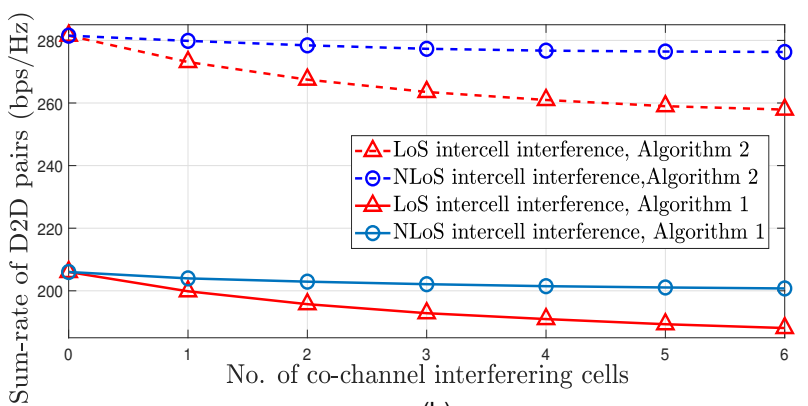

(b)

Fig. 10. Investigating the effect of intercell co-channel interference. Fig. (a) depicts the network structure, wherein central green cell serves D2D pairs, and cellular users from surrounding green cells impose co-channel intercell interference. Fig. (b) illustrates the performance of Algorithm 1 and Algorithm 2 for different number of co-channel interfering cells.

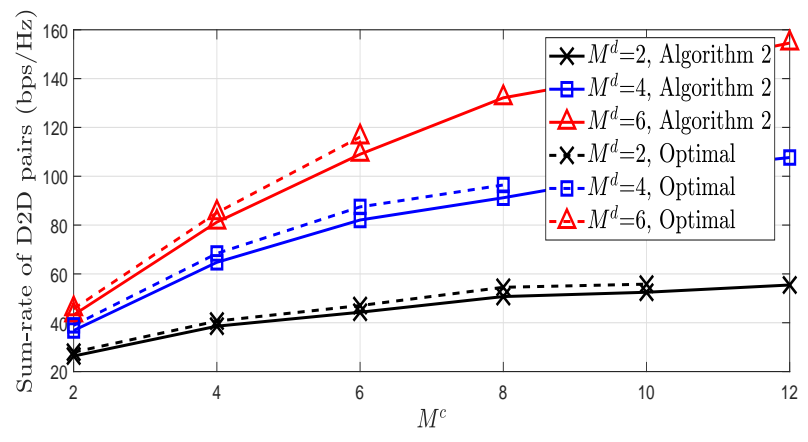

Fig. 11. Investigating the optimality of Algorithm 2.

5) Exploring the Effect of Intercell Interference: To investigate the effect of co-channel intercell interference, we consider a multi-cell cellular network according to Fig. VI-5. (a), wherein the central cell $C_{0}$ serves D2D pairs according to Algorithms 1 and 2, and a subset of surrounding cochannel interfering cells $C_{1}-C_{6}$ impose interference due to frequency reuse. Fig. VI-5. (b) shows the performance versus different number of co-channel interfering cells, considering LoS and NLoS intercell interferences It is observed that for both algorithms, NLoS intercell interference has little impact on the performance compared to LoS intercell interference. This is due to high NLoS path-loss exponent and high distance of interfering source from non-adjacent co-channel cells.

\footnotetext{
${ }^{5}$ By using the terms LoS and NLoS interferences, we mean that the channel between the interfering transmitter and the desired receiver is LoS and NLoS, respectively.
}

6) Optimality of Algorithm 2: Finally, we study the optimality of Algorithm 2 compared to globally optimal solution obtained by exhaustive search through all possible channel and link-type options. Fig. 11 illustrates this comparison versus different total number of users. Note that due to the exponential complexity of the exhaustive search, we are only able to present numerical results for small total number of D2D and cellular users. It is seen that the performance of our proposed algorithm is near-optimal for the evaluated total number of cellular and D2D users.

\section{CONCLUSION}

In this paper, we derived analytical closed form expressions of outage probability, ergodic capacity, and frame decoding error probability taking into account Nakagami-m and Rician fading channel models for terrestrial and aerial transmissions, respectively. The expressions were employed to devise efficient and ultra-reliable resource (subchannel, power, and linktype) management of D2D pairs and cellular users. Simulation results demonstrate the significance of aerial relays compared to ground relays in increasing the throughput of D2D pairs especially for distant D2D pairs. The framework can be extended to optimize the trajectory of UAV relays and a large scale network with multiple interferers. The characterization of frame decoding error probability can be extended under non-finite and non-quasi-static block regimes, and also, the proposed resource allocation can be extended for multi-hop UAV relaying in future works.

\section{APpendiX A: Proof of LEMMA 1}

Let $y$ and $x$ be unit mean random variables corresponding to the fading channels of the main LoS link $k$ and the interfering NLoS link $k^{\prime}$ respectively. From (8) we obtain (37) expressed at the top of next page wherein (a) is derived by expanding the modified Bessel function as $I_{0}(x)=\sum_{k=0}^{\infty} \frac{(x / 2)^{2 k}}{(k !)^{2}}$ ([40], Eq. 8.447), (b) and (d) are derived using the equality $\int e^{-c x} x^{m-1} d x=-e^{-c x} \frac{\Gamma(m)}{c^{m}} \sum_{k=0}^{m-1} \frac{(c x)^{k}}{k !}$, and (c) holds due to the equality $\int_{0}^{\infty} e^{-c x} x^{m-1} d x=\frac{\Gamma(m)}{c^{m}}$. Letting $\theta=$ $\frac{\alpha(K+1)}{m+\alpha(K+1)}$ results in

$$
\begin{aligned}
& O_{L, N}(\alpha)=1-\frac{\frac{m^{m}}{\Gamma(m)} e^{-K} \sum_{j=0}^{\infty} \frac{K^{j}}{j !} \frac{d^{m-1}}{d \theta^{m-1}}\left[\sum_{k=0}^{j} \theta^{k+m-1}\right]}{(m+\alpha(K+1))^{m}} \\
= & 1-\frac{m^{m} e^{-K}}{\Gamma(m)(m+\alpha(K+1))^{m}} \frac{d^{m-1}}{d \theta^{m-1}}\left[\frac{\theta^{m-1}}{1-\theta}\left(e^{K}-\theta e^{K \theta}\right)\right] .
\end{aligned}
$$

\section{APPENDix B: Proof OF LEMMA 3}

Let $y$ and $x$ be unit mean Gamma distributed random variables with shape factors $m$ and $m^{\prime}$ corresponding to the desired link $k$ and interfering link $k^{\prime}$ respectively. We have

$$
\begin{aligned}
& O_{N, N}(\alpha)=\operatorname{Pr}\{y<\alpha x\}=\int_{0}^{\infty} \int_{0}^{\alpha x} f_{G}\left(x, m^{\prime}\right) f_{G}(y, m) d y d x \\
& =\frac{m^{m} m^{\prime m^{\prime}}}{\Gamma(m) \Gamma\left(m^{\prime}\right)} \int_{0}^{\infty} x^{m^{\prime}-1} e^{-m^{\prime} x} \int_{0}^{\alpha x} y^{m-1} e^{-m y} d y d x
\end{aligned}
$$


$O_{L, N}(\alpha)=\operatorname{Pr}\{y<\alpha x\}=\int_{0}^{\infty} \int_{0}^{\alpha x} f_{G}(x, m) f_{R}(y, K) d y d x$
$=\frac{m^{m}(K+1)}{\Gamma(m) e^{K}} \times \int_{0}^{\infty} \int_{0}^{\alpha x} x^{m-1} e^{-m x-(K+1) y} I_{0}(\sqrt{4 K(K+1) y}) d y d x$
$\stackrel{(a)}{=} \frac{m^{m}(K+1)}{\Gamma(m) e^{K}} \times \int_{0}^{\infty} x^{m-1} e^{-m x} \int_{0}^{\alpha x} e^{-(K+1) y} \sum_{j=0}^{\infty} \frac{(K(K+1))^{j} y^{j}}{(j !)^{2}} d y d x$
$=\frac{m^{m}(K+1)}{\Gamma(m) e^{K}} \times \sum_{j=0}^{\infty} \frac{(K(K+1))^{j}}{(j !)^{2}} \int_{0}^{\infty} x^{m-1} e^{-m x} \int_{0}^{\alpha x} e^{-(K+1) y} y^{j} d y d x$
$\stackrel{(b)}{=} \frac{m^{m}(K+1)}{\Gamma(m) e^{K}} \sum_{j=0}^{\infty} \frac{(K(K+1))^{j}}{(j !)^{2}} \times \int_{0}^{\infty} x^{m-1} e^{-m x}\left[-\frac{e^{-(K+1) y} j !}{(K+1)^{j+1}} \sum_{k=0}^{j} \frac{((K+1) y)^{k}}{k !}\right]_{0}^{\alpha x} d x$
$\stackrel{(c)}{=} \frac{m^{m}}{\Gamma(m) e^{K}} \sum_{j=0}^{\infty} \frac{(K)^{j}}{j !} \times\left(\frac{\Gamma(m)}{m^{m}}-\sum_{k=0}^{j} \frac{(\alpha(K+1))^{k}}{k !} \int_{0}^{\infty} e^{-x(m+\alpha(K+1))} x^{k+m-1} d x\right)^{m} e^{-K}$
$\stackrel{(d)}{=} 1-\frac{m^{m} e^{-K}}{\Gamma(m)} \sum_{j=0}^{\infty} \frac{K^{j}}{j !} \sum_{k=0}^{j} \frac{(\alpha(K+1))^{k} \Gamma(k+m)}{k !(m+\alpha(K+1))^{k+m}}=1-\frac{K^{j}}{(m+\alpha(K+1))^{m}} \sum_{j=0}^{j} \frac{\alpha}{j !} \sum_{k=0}^{j}\left(\frac{\alpha(K+1)}{m+\alpha(K+1)}\right)^{k} \frac{\Gamma(k+m)}{k !}$

$\stackrel{(a)}{=} 1-\frac{m^{\prime m^{\prime}}}{\Gamma\left(m^{\prime}\right)} \int_{0}^{\infty} x^{m^{\prime}-1} e^{-x\left(m^{\prime}+m \alpha\right)} \sum_{k=0}^{m-1} \frac{(m \alpha x)^{k}}{k !} d x$

$\stackrel{(b)}{=} 1-\frac{m^{\prime m^{\prime}}}{\Gamma\left(m^{\prime}\right)} \sum_{k=0}^{m-1} \frac{(m \alpha)^{k} \Gamma\left(k+m^{\prime}\right)}{k !\left(m^{\prime}+m \alpha\right)^{k+m^{\prime}}}$,

where (a) and (b) are derived using the equalities $\int e^{-c x} x^{m-1} d x=-e^{-c x} \frac{\Gamma(m)}{c^{m}} \sum_{k=0}^{m-1} \frac{(c x)^{k}}{k !} \quad$ and $\int_{0}^{\infty} e^{-c x} x^{m-1} d x=\frac{\Gamma(m)}{c^{m}}$, respectively.

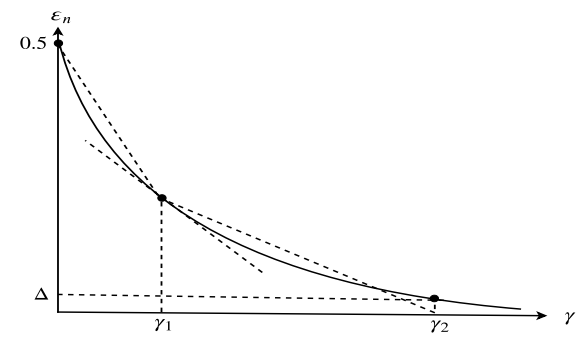

\section{Appendix C: Proof of Theorem 1}

We can verify that $u(\gamma)$ is monotonically increasing and hence $Q(u(\gamma))$ is a monotonically decreasing function of $\gamma$. We can also show that $\lim _{\gamma \rightarrow 0} \varepsilon_{n}(\gamma)=Q(0)=0.5$, and $\lim _{\gamma \rightarrow \infty} \varepsilon_{n}(\gamma)=0$. Fig. 12 shows a 2-level piece-wise linear approximation of (24). To achieve a better approximation, we can extend that to $L$-level piece-wise linear approximation, and estimate $\varepsilon_{n}(\gamma)$ as:

$$
\varepsilon_{n}(\gamma) \approx \begin{cases}-\omega_{i} \gamma+\omega_{i} \gamma_{i}+0.5\left(1-\frac{i}{L}\right), & \text { if } \gamma_{i-1} \leq \gamma<\gamma_{i}, \\ 0, & \text { if } \gamma \geq \gamma_{L},\end{cases}
$$

where $\omega_{i}=\left(\varepsilon_{n}\left(\gamma_{i-1}\right)-\varepsilon_{n}\left(\gamma_{i}\right)\right) /\left(\gamma_{i}-\gamma_{i-1}\right)=\frac{0.5 / L}{\gamma_{i}-\gamma_{i-1}}, \gamma_{i}=$ $\varepsilon^{-1}(0.5(1-i / L))$ for $0 \leq i \leq L-1$, and $\gamma_{L}=\varepsilon_{n}^{-1}(\Delta)$, in which $\Delta \ll 0.5 / L$ is a small value constant.

The average decoding error probability is then obtained as follows:

$$
\begin{aligned}
& \bar{\varepsilon}_{n}=\mathbb{E}\left\{\varepsilon_{n}(\gamma)\right\}=\int_{0}^{\infty} f_{\gamma}(\gamma) \varepsilon_{n}(\gamma) d \gamma=K_{0}+\sum_{i=1}^{L} \omega_{i} \int_{\gamma_{i-1}}^{\gamma_{i}} F_{\gamma}(\gamma) d \gamma \\
& =K_{0}+\sum_{i=1}^{L-1}\left(\omega_{i-1}-\omega_{i}\right) H\left(\zeta_{j, i}, \gamma_{i}\right)+\omega_{L} H\left(\zeta_{j, i}, \gamma_{L}\right)
\end{aligned}
$$

where

$$
H(\alpha, \gamma)=\int_{0}^{\gamma} F_{\gamma}(\gamma) d \gamma=\int_{0}^{\gamma} O^{c}(\alpha \gamma) d \gamma,
$$

and $K_{0}$ is obtained as follows:

$$
\begin{aligned}
& K_{0}=\sum_{i=1}^{L}-\omega_{i}\left(\gamma_{i} F\left(\gamma_{i}\right)-\gamma_{i-1} F\left(\gamma_{i-1}\right)\right) \\
& \quad+\left(\omega_{i} \gamma_{i}+0.5(1-i / L)\right)\left(F\left(\gamma_{i}\right)-F\left(\gamma_{i-1}\right)\right) \\
& \stackrel{(a)}{=} \sum_{i=1}^{L} \frac{0.5}{L} F\left(\gamma_{i-1}\right)+0.5(1-i / L)\left(F\left(\gamma_{i}\right)-F\left(\gamma_{i-1}\right)\right) \\
& =0.5 \sum_{i=1}^{L} F\left(\gamma_{i}\right)(1-i / L)-F\left(\gamma_{i-1}\right)(1-(i-1) / L) \\
& =0.5\left(F\left(\gamma_{0}\right)+F\left(\gamma_{L}\right)(1-L / L)\right)=0,
\end{aligned}
$$

where (a) follows from $\omega_{i}=\frac{0.5 / L}{\gamma_{i}-\gamma_{i-1}}$. For the NLoS interference, from (39), (17), and (18b) we calculate $H(\alpha, \gamma)$ for the cellular user as follows:

$$
\begin{aligned}
H_{N}(\alpha, \gamma) & =\int_{0}^{\gamma} O^{c}(\alpha \gamma) d \gamma=\int_{0}^{\gamma} O_{N, N}(\alpha \gamma) d \gamma \\
= & \gamma-\frac{m^{\prime m^{\prime}}}{\Gamma\left(m^{\prime}\right)} \sum_{k=0}^{m-1} \int_{0}^{\gamma} \frac{(m \alpha \gamma)^{k} \Gamma\left(k+m^{\prime}\right)}{k !\left(m^{\prime}+m \alpha \gamma\right)^{k+m^{\prime}}} d \gamma \\
= & \gamma-\frac{m^{\prime m^{\prime}}}{\Gamma\left(m^{\prime}\right)} \sum_{k=0}^{m-1} \frac{\Gamma\left(k+m^{\prime}\right)(m \alpha)^{k} \gamma^{k+1}}{(k+1) !\left(m^{\prime}\right)^{k+m^{\prime}}}
\end{aligned}
$$




$$
\times{ }_{2} F_{1}\left(k+1, k+m^{\prime}, k+2,-\frac{m \alpha \gamma}{m^{\prime}}\right) .
$$

Similarly, for the LoS interference scenario, by using from (39), (16) and (18a) we have

$$
\begin{aligned}
& H_{L}(\alpha, \gamma)=\int_{0}^{\gamma} O^{c}(\alpha \gamma) d \gamma=\int_{0}^{\gamma} O_{N, L}(\alpha \gamma) d \gamma \\
& =\int_{0}^{\gamma}\left(1-O_{L, N}(1 / \alpha \gamma)\right) d \gamma \\
& =\frac{1}{\Gamma(m)} e^{-K} \sum_{j=0}^{\infty} \frac{K^{j}}{j !} \sum_{k=0}^{j} \frac{\Gamma(k+m)(K+1)^{k}}{k !} \\
& \quad \times \int_{0}^{\gamma} \frac{(m \alpha \gamma)^{m}}{(K+1+m \alpha \gamma)^{k+m}} d \gamma \\
& =\frac{e^{-K}(m \alpha)^{m} \gamma^{m+1}}{\Gamma(m)(K+1)^{m}(m+1)} \sum_{j=0}^{\infty} \frac{K^{j}}{j !} \sum_{k=0}^{j} \frac{\Gamma(k+m)}{k !} \\
& \quad \times{ }_{2} F_{1}\left(m+1, m+k, m+2,-\frac{m \alpha \gamma}{K+1}\right) .
\end{aligned}
$$

\section{REFERENCES}

[1] M. Höyhtyä, O. Apilo, and M. Lasanen, "Review of latest advances in 3GPP standardization: D2D communication in 5G systems and its energy consumption models," Future Internet, vol. 10, no. 1, p. 3, 2018

[2] P. Osti, P. Lassila, and S. Aalto, "Performance of D2D underlay and overlay for elastic traffic," in Proc. of the 19th ACM Intl. Conf. on Modeling, Analysis and Simulation of Wireless and Mobile Systems. ACM, 2016, pp. 270-277.

[3] 3GPP TE 22.803 V1. 0.0, "3rd generation partnership project; Technical specification group SA; Feasibility study for proximity services (ProSe)(Release 12)," 2012.

[4] 3GPP Release 13. [Online]. Available: https://www.3gpp.org/release-13

[5] W. Sang, et al., "A survey of capacity in cooperative relay networks," in 2011 Global Mobile Congress. IEEE, 2011.

[6] R. Kumar and A. Hossain, "Survey on half-and full-duplex relay based cooperative communications and its potential challenges and open issues using markov chains," IET Commun., vol. 13, no. 11, pp. 1537-1550, 2019.

[7] L. Zhang, H. Zhao, S. Hou, Z. Zhao, H. Xu, X. Wu, Q. Wu, and R. Zhang, "A Survey on 5G millimeter wave communications for UAVassisted wireless networks," IEEE Access, 2019.

[8] B. Li, Z. Fei, and Y. Zhang, "UAV communications for 5G and beyond: Recent advances and future trends," IEEE Internet of Things Journal, vol. 6, no. 2, pp. 2241-2263, 2018.

[9] P. Zhou, X. Fang, Y. Fang, R. He, Y. Long, and G. Huang, "Beam management and self-healing for mmWave UAV mesh networks," IEEE Transactions on Vehicular Technology, vol. 68, no. 2, pp. 1718-1732, 2018.

[10] Y. Huo, F. Lu, F. Wu, and X. Dong, "Multi-beam multi-stream communications for $5 \mathrm{G}$ and beyond mobile user equipment and UAV proof of concept designs," in IEEE Vehicular Technology Conference, 2019.

[11] W. Khawaja, O. Ozdemir, and I. Guvenc, "UAV air-to-ground channel characterization for mmWave systems," in 2017 IEEE 86th Vehicular Technology Conference (VTC-Fall). IEEE, 2017, pp. 1-5.

[12] H. Wang, J. Wang, G. Ding, Y. Chen, and Z. Han, "Spectrum sharing planning for full-duplex UAV relaying systems with underlaid D2D communications," IEEE Journal on Selected Areas in Commun., vol. 36, no. 9, pp. 1986-1999, 2018.

[13] B. Ma, H. Shah-Mansouri, and V. W. Wong, "Full-duplex relaying for D2D communication in millimeter wave-based $5 \mathrm{G}$ networks," IEEE Trans. on Wireless Commun., vol. 17, no. 7, pp. 4417-4431, 2018.

[14] M. Monemi, M. Rasti, and E. Hossain, "On characterization of feasible interference regions in cognitive radio networks," IEEE Trans. on Commun., vol. 64, no. 2, pp. 511-524, 2016.

[15] S. Sekander, H. Tabassum, and E. Hossain, "Decoupled uplink-downlink user association in multi-tier full-duplex cellular networks: A two-sided matching game," IEEE Trans. on Mobile Computing, vol. 16, no. 10, pp. 2778-2791, 2016.
[16] Y. Chen, et al., "Resource allocation for device-to-device communications underlaying heterogeneous cellular networks using coalitional games," IEEE Trans. on Wireless Commun., vol. 17, no. 6, pp. 41634176, 2018.

[17] S. Kusaladharma and C. Tellambura, "Interference and outage in random D2D networks under millimeter wave channels," in 2017 IEEE Intl. Conf. on Commun. (ICC). IEEE, 2017, pp. 1-7.

[18] C. She, C. Liu, T. Q. Quek, C. Yang, and Y. Li, "Ultra-reliable and low-latency communications in unmanned aerial vehicle communication systems," IEEE Transactions on Communications, vol. 67, no. 5, pp. 3768-3781, 2019.

[19] J. Li, X. Li, A. Wang, and N. Ye, "Performance Analysis for Downlink MIMO-NOMA in Millimeter Wave Cellular Network with D2D Communications," Wireless Commun. and Mobile Computing, vol. 2019, 2019.

[20] G. D. Swetha and G. R. Murthy, "Selective overlay mode operation for D2D communication in dense 5G cellular networks," in 2017 IEEE Symp. on Computers and Commun. (ISCC). IEEE, 2017, pp. 704-709.

[21] D. Mishra, P. Lohan, and L. N. Devi, "Coverage-constrained utility maximization of UAV," in ICC 2019-2019 IEEE Intl. Conf. on Commun. (ICC). IEEE, 2019, pp. 1-6.

[22] L. Sboui, H. Ghazzai, Z. Rezki, and M.-S. Alouini, "Energy-efficient power allocation for UAV cognitive radio systems," in 2017 IEEE 86th Veh. Tech. Conf. (VTC-Fall). IEEE, 2017, pp. 1-5.

[23] D. Della Penda, N. Nomikos, T. Charalambous, and M. Johansson, "Minimum power scheduling under Rician fading in full-duplex relayassisted D2D communication," in 2017 IEEE Globecom Workshops (GC Wkshps). IEEE, 2017, pp. 1-6.

[24] J. Baek, S. I. Han, and Y. Han, "Optimal resource allocation for nonorthogonal transmission in UAV relay systems," IEEE Wireless Commun. Letters, vol. 7, no. 3, pp. 356-359, 2017.

[25] Y. Wang, M. Chen, N. Huang, Z. Yang, and Y. Pan, "Joint power and channel allocation for D2D underlaying cellular networks with Rician fading," IEEE Commun. Letters, vol. 22, no. 12, pp. 2615-2618, 2018.

[26] M. K. Mishra and A. Trivedi, "Spectral efficiency and deployment cost efficiency analysis of mmW/UHF based cellular network," IEEE Trans. on Veh. Tech., 2019.

[27] T. Shafique, H. Tabassum, and E. Hossain, "End-to-end energyefficiency and reliability of uav-assisted wireless data ferrying," IEEE Transactions on Communications, vol. 68, no. 3, pp. 1822-1837, 2019.

[28] Y. Polyanskiy, H. V. Poor, and S. Verdú, "Channel coding rate in the finite blocklength regime," IEEE Trans. on Inf. Theory, vol. 56, no. 5, p. $2307,2010$.

[29] A. Annamalai, C. Tellambura, and V. K. Bhargava, "Simple and accurate methods for outage analysis in cellular mobile radio systems-a unified approach," IEEE Transactions on Communications, vol. 49, no. 2, pp. 303-316, 2001.

[30] A. Al-Hourani, S. Kandeepan, and S. Lardner, "Optimal LAP altitude for maximum coverage," IEEE Wireless Commun. Letters, vol. 3, no. 6, pp. 569-572, 2014.

[31] M. Mozaffari, W. Saad, M. Bennis, and M. Debbah, "Unmanned aerial vehicle with underlaid device-to-device communications: Performance and tradeoffs," IEEE Trans. on Wireless Commun., vol. 15, no. 6, pp. 3949-3963, 2016.

[32] 3GPP, "Tr 36.828 (v11.0.0): Further enhancements to LTE time division duplex (TDD) for downlink-uplink (DL-UL) interference management and traffic adaptation," 2016.

[33] T. Riihonen, S. Werner, and R. Wichman, "Hybrid full-duplex/halfduplex relaying with transmit power adaptation," IEEE Transactions on Wireless Communications, vol. 10, no. 9, pp. 3074-3085, 2011.

[34] N. Khoolenjani and K. Khorshidian, "On the ratio of Rice random variables," 2009.

[35] W. Yang, G. Durisi, T. Koch, and Y. Polyanskiy, "Quasi-static multipleantenna fading channels at finite blocklength," IEEE Trans. on Inf. Theory, vol. 60, no. 7, pp. 4232-4265, 2014.

[36] D. B. West et al., Introduction to graph theory. Prentice hall Upper Saddle River, NJ, 1996, vol. 2.

[37] M. R. Akdeniz, Y. Liu, M. K. Samimi, S. Sun, S. Rangan, T. S. Rappaport, and E. Erkip, "Millimeter wave channel modeling and cellular capacity evaluation," IEEE journal on selected areas in communications, vol. 32 , no. 6, pp. 1164-1179, 2014.

[38] R. K. Martin and R. Thomas, "Algorithms and bounds for estimating location, directionality, and environmental parameters of primary spectrum users," IEEE Trans. on Wireless Commun., vol. 8, no. 11, pp. $5692-5701,2009$. 
[39] J. Werner, et al., "Performance and Cramer-Rao bounds for DoA/RSS estimation and transmitter localization using sectorized antennas," IEEE Trans. on Veh. Tech., vol. 65, no. 5, pp. 3255-3270, 2015.

[40] I. S. Gradshteyn and I. M. Ryzhik, Table of integrals, series, and products. Academic press, 2014.

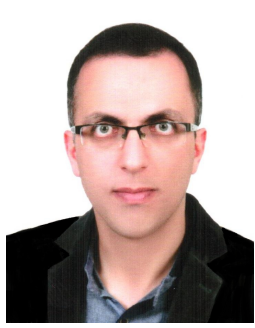

Mehdi Monemi Mehdi Monemi received the B.Sc., M.Sc., and Ph.D. degrees all in electrical and computer engineering from Shiraz University, Shiraz, Iran, and Tarbiat Modares University, Tehran, Iran, and Shiraz University, Shiraz, Iran in 2001, 2003 and 2014 respectively. After receiving his Ph.D, he has been working as the project manager in several companies, and is currently an assistant professor in the Department of Electrical Engineering, Salman Farsi University of Kazerun, Kazerun, Iran. He was a visiting researcher in the Department of Electrical and Computer Engineering, York university, Toronto, Canada from June 2019 to September 2019, Iran. His current research interests include resource allocation in wireless networks, and traffic engineering in computer networks.

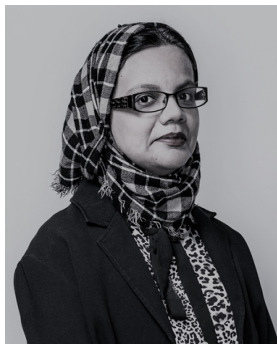

Hina Tabassum (SM'17) Hina Tabassum is currently an Assistant Professor at the Lassonde School of Engineering, York University, Canada. Prior to that, she was a postdoctoral research associate at the Department of Electrical and Computer Engineering, University of Manitoba, Canada. She received her $\mathrm{PhD}$ degree from King Abdullah University of Science and Technology (KAUST). She is a Senior member of IEEE and registered Professional Engineer in the province of Ontario, Canada. She has been recognized as an Exemplary Reviewer (Top $2 \%$ of all reviewers) by IEEE Transactions on Communications in 2015, 2016, 2017, and 2019. Currently, she is serving as an Associate Editor in IEEE Communications Letters and IEEE Open Journal of Communications Society. Her research interests include stochastic modeling and optimization of wireless networks including vehicular, aerial, and satellite networks, millimeter and terahertz communication networks, software-defined networking and virtualized resource allocation in wireless networks. 